\title{
Risk-Promoting Effects of Reward-Paired Cues in Human Sign- and Goal-Trackers
}

Mariya V. Cherkasova, $\mathrm{PhD}^{1,2 \#}$, Eve Limbrick-Oldfield, $\mathrm{PhD}^{1}$, Luke Clark, $\mathrm{PhD}^{1,2}$, Jason J.S.

Barton, MD, PhD ${ }^{3}$, A. Jon Stoessl, CM, MD, FRCPC, FAAN, FCAHS ${ }^{2,3}$, Catharine A.

Winstanley, $\mathrm{PhD}^{1,2}$

${ }^{1}$ Department of Psychology, University of British Columbia, Vancouver, British Columbia, Canada

${ }^{2}$ Djavad Mowafaghian Centre for Brain Health, University of British Columbia, Vancouver, British Columbia, Canada

${ }^{3}$ Department of Medicine, Division of Neurology, University of British Columbia, Vancouver, British Columbia, Canada

${ }^{4}$ Department of Ophthalmology, University of British Columbia, Vancouver, British Columbia, Canada

${ }^{\#}$ Current Address: Department of Psychology, West Virginia University, Morgantown, West Virginia, USA

Corresponding author:

Dr. Catharine A. Winstanley or Dr. Mariya V. Cherkasova, Djavad Mowafaghian Centre for Brain Health University of British Columbia

2215 Wesbrook Mall

Vancouver, BC, V6T 1Z3, Canada

E-mail: cwinstanley@psych.ubc.ca or mariya.cherkasova@ mail.wvu.edu

Manuscript word count: 5678

Abstract: 225

Figures: 3

Tables: 0

Running title: Risk-promoting effects of cues and sign-tracking 
Risk-promoting effects of cues and sign-tracking

\section{ABSTRACT}

The incentive sensitization theory of addiction proposes that through repeated associations with addictive rewards, addiction-related stimuli acquire a disproportionately powerful motivational pull on behaviour. Animal research suggests trait-like individual variation in the degree of incentive salience attribution to reward-predictive cues, defined phenotypically as sign-tracking (high) and goal-tracking (low incentive salience attribution). While these phenotypes have been linked to addiction features in rodents, their translational validity has been little studied. Here, we examined whether sign- and goal-tracking in healthy human volunteers modulates the effects of reward-paired cues on cost-benefit decision making. Sign-tracking was measured in a Pavlovian conditioning paradigm as the amount of eye gaze fixation on the reward-predictive cue versus the location of impending reward delivery. In Study 1 (Cherkasova et al, 2018), participants were randomly assigned to perform a two-choice lottery task in which rewards were either accompanied (cued, $n=63$ ) or unaccompanied (uncued, $n=68$ ) by money images and casino jingles. In Study 2, participants $(n=58)$ performed cued and uncued versions of the task in a within-subjects design. Across both studies, cues promoted riskier choice, and both studies yielded evidence of goal-tracking being associated with greater risk-promoting effects of cues. These findings are at odds with the notion of sign-trackers being preferentially susceptible to the influence of reward cues on behavior and point to the role of mechanisms besides incentive salience in mediating such influences. 
Risk-promoting effects of cues and sign-tracking

\section{Introduction}

Cue-reactivity refers to the amplified subjective, behavioural, psychophysiological or neural response to addiction-related stimuli in individuals with substance-related and addictive disorders (Childress et al., 1993). Cue reactivity has been observed in a variety of substance and behavioural addictions (Carter \& Tiffany, 1999)(Norberg, Kavanagh, Olivier, \& Lyras, 2016)(Limbrick-Oldfield et al., 2017)(Boswell, Kober, Haven, \& Haven, 2018)(Goudriaan, De Ruiter, Van Den Brink, Oosterlaan, \& Veltman, 2010) and is thought to play an important role in addiction course and outcome. For example, attentional biases and neural reactivity to addictionrelated cues have been found to predict treatment outcome and duration of abstinence (Field \& Cox, 2008)(Jasinska, Stein, Kaiser, Naumer, \& Yalachkov, 2014)(MacNiven et al., 2019).

The incentive sensitization theory of addiction offers a mechanistic account of cue reactivity. The theory proposes that through repeated associations with addictive rewards, addiction-related stimuli acquire incentive salience, and thereby exert a disproportionately powerful motivational pull on behaviour (Robinson \& Berridge, 1993). Sensitization of the dopamine system's response to both rewards (such as drugs) and reward-paired cues is proposed as the underlying neurobiological mechanism of these motivational effects. However, it remains unclear whether cue reactivity observed in addictive disorders is solely a consequence of the powerful cue-reward associations established in the course of the addition process, or if a preexisting trait-like propensity to forming, or being influenced by, cue-reward association may contribute to this process.

Animal research suggests important individual variation in the propensity to attribute incentive salience to reward-predictive cues (Flagel, Akil, \& Robinson, 2009)(Meyer et al., 2012). During Pavlovian conditioning, some animals (termed sign-trackers, ST) display 
Risk-promoting effects of cues and sign-tracking

appetitive approach towards reward predictive neutral cues (such as a lever extended from the wall of an operant chamber) as if these cues themselves have acquired incentive motivational properties, whereas other animals (termed goal-trackers, GT) respond to the same cues as a signal to approach the location of impending reward delivery (the food tray). In rodent models, these phenotypes have been differentially linked to addiction-related features: sign-trackers work harder for cocaine on a progressive ratio task (Saunders \& Robinson, 2011) and are more prone to cue-induced cocaine-seeking in extinction (Yager \& Robinson, 2013), as well as cue- and drug-induced reinstatement of cocaine seeking (Saunders \& Robinson, 2010)(Saunders \& Robinson, 2011)(Saunders \& Robinson, 2013). By contrast, goal-trackers exhibit greater contextinduced reinstatement of cocaine seeking and context-conditioned hyperactivity (Saunders, O’Donnell, Aurbach, \& Robinson, 2014). Based on this pattern of data, it was proposed that STs are particularly sensitive to discrete and localizable reward-predictive cues (e.g. lever), whereas GTs are more sensitive to contextual cues (e.g. floor texture, illumination, odor of the operant chamber) (Robinson, Yager, Cogan, \& Saunders, 2014). Some initial evidence suggests that similar phenotypes exist in humans, (Garofalo \& di Pellegrino, 2015)(Joyner, Gearhardt, \& Flagel, 2018)(Wardle, Lopez-Gamundi, \& Flagel, 2018)(Schad et al., 2020), though few studies have addressed their translational validity.

One candidate mechanism through which sensitivity to cues may translate into addictive behaviour is through the influence cues have on cost-benefit decision making; salient rewardpaired cues promote riskier choice in both rodents (Barrus \& Winstanley, 2016) (Ferland et al., 2019)(Adams, Barkus, Ferland, Sharp, \& Winstanley, 2017) and humans (Cherkasova et al., 2018)(Ludvig, Madan, \& Spetch, 2015)(Spetch, Madan, Liu, \& Ludvig, 2020). Our goal was therefore to examine whether sign- and/or goal-tracking in healthy human volunteers was 
associated with the risk-promoting effects of reward-paired cues. Across two experiments, we classified participants as STs and GTs using an eye-tracking procedure adapted from the first published human study examining the translational validity of sign-tracking (Garofalo \& di Pellegrino, 2015). In the first study, we evaluated the effects of reward cues on risky choice using a separate procedure, a two-choice lottery task, with the presence of cues manipulated in a between-subject design. In a second study, we aimed to replicate the risk-promoting effect of cues observed in Study 1 using a within-subject design, and we further manipulated the timing of the cues in the risky choice task. In Study 1, cues only accompanied reward delivery, consistent with the rodent paradigm that inspired the human study (Barrus \& Winstanley, 2016) and thus had little reward-predictive significance. In Study 2, visual reward cues were also present at the time of choice and could theoretically predict rewards or incentivize reward-seeking choices. Based on the preponderance of animal findings linking sign-tracking with addiction features in animal models, we hypothesized that sign-trackers (as defined using eye-tracking) would be more susceptible to the biasing effects of cues on cost-benefit decision making.

\section{Study 1}

\section{Materials and Methods}

\section{Participants}

The results of the two-choice lottery task from Study 1 has previously been reported (Cherkasova et al., 2018), which demonstrated that reported that reward-paired cues promoted riskier choice in these participants. Briefly, 131 community volunteers took part in Study 1 (males: $\mathrm{n}=53$, females: $\mathrm{n}=78$, mean age $=25.65 \pm 8.28$ ). The sample size was chosen assuming a medium effect size for the effect of reward cues on decision making in a betweensubject design. Self-report data were collected regarding their medication and substance use via 
Module E from the Structured Clinical Interview for DSM-IV Axis I disorders (SCID-IV) (First, Spitzer, Gibbon, \& Williams, 1999). Five participants reported ongoing use of psychotropic medications: escitalopram for major depression $(n=1)$ and stimulants for attention deficit hyperactivity disorder $(n=4)$. Two participants met criteria for substance dependence. Because excluding the data from these participants did not change the significance of the findings, and their data did not display any unusual patterns (e.g. extreme values) we report the results from the entire sample.

Participants were excluded for being at-risk gamblers as indicated by scores $\geq 3$ on the Problem Gambling Severity Index (Wynne, 2003) and for having visual or hearing impairments that would compromise their ability to perform the task or appropriately perceive the sensory stimuli. Studies were conducted in accordance with institutional guidelines and the Declaration of Helsinki and were approved by the Research Ethics Board of the University of British Columbia. Participants gave written informed consent. Compensation corresponded to the bonus amounts earned on the tasks.

\section{Procedure}

Participants were randomly assigned to two groups. Group $1(n=63)$ performed the twochoice lottery Vancouver Gambling Task (VGT) without the sensory reward cues (henceforth 'uncued'); Group $2(\mathrm{n}=68)$ performed the VGT with the sensory reward cues ('cued'). Thus, the effect of cues on decision making was evaluated between subjects. The two groups did not differ significantly in terms of age or gender composition $\left(\mathrm{p}_{\mathrm{s}} \geq 0.62\right)$. As described in (Cherkasova et al., 2018), participants also performed cued and uncued versions of the Iowa Gambling Task, not reported in this paper, with the order of the decision-making tasks randomized across 
participants. The decision-making tasks were followed by the Pavlovian Instrumental Transfer paradigm modelled after (Garofalo \& di Pellegrino, 2015), with sign-tracking measured during the Pavlovian phase.

All tasks were accompanied by eye tracking using the EyeLink 1000 infrared pupil tracker with a sampling rate of $1000 \mathrm{~Hz}$ and resolution of $0.01^{\circ}$ of visual angle (SR Research Ltd., Mississauga, Ontario), see Supplement for details. Prior to each task, a 5-point calibration was performed. Stimulus presentation and data collection were controlled via scripts developed in Experiment Builder (the eye tracker's proprietary software).

\section{Tasks}

\section{Vancouver Gambling Task}

This two-choice lottery task assesses willingness to take risks at different combinations of reward probability and magnitude (Figure 1A). Participants made a choice between two options on every trial. One option featured a larger but less probable gain, while the other featured a smaller and more probable gain. The odds of winning for the two options always summed to 100\%: $20 \%$ vs. $80 \%, 30 \%$ vs. $70 \%, 40 \%$ vs. $60 \%$. There were 10 unique option pairs, each repeated 10 times for the total of 100 trials. These 10 pairs formed a continuum of relative expected values (probability x magnitude, EV) of the options, ranging from pairs that highly favored the 'safer' choice (i.e. the smaller but more probable gain) to pairs that highly favored the 'riskier' choice (i.e. the larger but less probable gain). Thus, each pair was associated with a unique Expected Value Difference Ratio (EV-ratio) calculated as $\left[\mathrm{EV}_{(\mathrm{safe})}-\right.$ $\left.\mathrm{EV}_{(\text {risky) }}\right] /$ mean( $\left.\mathrm{EV}_{(\text {(safe) }}, \mathrm{EV}_{\text {(risky) }}\right)$ as per (Cherkasova et al., 2018). 
Risk-promoting effects of cues and sign-tracking

On each trial, the participant either won the reward of the chosen gamble or received nothing: no losses occurred. For the uncued VGT gain feedback was delivered using numerals, without sound accompaniment. For the cued VGT, the gains were represented by images of coins accompanied by casino jingles. These visual and auditory cues scaled in sensory intensity and complexity with gain magnitude; see (Cherkasova et al., 2018) for a detailed description of the audiovisual cues. To ensure that participants understood the task, they were given 5 practice trials before the start of the 100 test trials. At the end of every 20 trials, they were offered a break.

\section{Pavlovian Conditioning}

Sign-tracking and goal-tracking responses were assessed in a Pavlovian conditioning task embedded in a Pavlovian Instrumental Transfer (PIT) procedure that consisted of three stages: 1) instrumental learning, 2) Pavlovian conditioning, and 3) the transfer stage. The task was modelled after (Garofalo \& di Pellegrino, 2015). Because only the Pavlovian task was used to measure the sign-/ goal-tracking, we focus on the Pavlovian task in the Methods and Results. Methods and results for the instrumental and PIT tasks are in the Supplement.

In the Pavlovian stage (Figure 1C) that we focus on here, participants were instructed that the computer would determine when they received rewards; their role was to discover them by pressing the spacebar. After a variable inter-trial interval (500-4000 ms) displaying a black background with four empty squares $\left(4 \mathrm{~cm}^{2}\right)$ positioned at top center, bottom center, left center and right center of the screen, participants were presented with one of two conditioned stimuli inside the top square: the CS+ predicted a reward in $80 \%$ of trials, and the CS- was never associated with reward. These visual cues were fractal images, which differed in colour and 
shape, with the predictive value of the visual cues randomized across participants. The CS remained on the screen for the duration of $5000 \mathrm{~ms}$, during which the sign-tracking / goaltracking conditioned response (CR) was measured as the amount of gaze fixation on the CS+ versus the location of the impending reward delivery in the square at the bottom of the screen. After 5000ms, the CS was removed from the display, and the square at the bottom of the display turned white to signal to the participants that the outcome was available, and a spacebar response was required for it to be revealed. Pressing the spacebar triggered the feedback display, during which the reward (a $25 \phi$ coin) or non-reward (an empty circle of the same hue and luminance) and the fractal image that predicted it remained together on the screen for $1000 \mathrm{~ms}$. The task consisted of 40 trials (20 per CS condition). At the end of the task participants were asked to rate their liking of the fractal images used as CS+ and CS- on a 5-point Likert scale.

\section{Analyses}

Statistical analyses were performed using R and Matlab 2019a. Analysis code is available online (https://osf.io/9b34v/; https://github.com/mcherkasova/STGT_VGT). Linear mixed effects models were fitted using the lme4 package (Bates, Mächler, Bolker, \& Walker, 2015). Model assumptions were checked visually, and no violations identified. The influence of each participant on the models was assessed by considering residuals, Cook's distances and standardized DF-Betas for each participant, as well as testing for changes in significance for the predictors of interest with the removal of each influential participant. In the case of multilevel models, this was accomplished using the influence.ME package (Nieuwenhuis, te Grotenhuis, \& Pelzer, 2012). For influential cases that altered the predictors' significance, we checked for the possibility of error in data collection or entry and considered whether there was any objective reason to exclude these participants. Although we found no objective reasons to exclude any 
participants, there was one influential participant (Study 1) that changed the statistical significance / non-significance in the decision-making analyses, albeit without substantively changing the study's conclusions. We report the results excluding this participant in the Supplement. For the models below, we report statistically significant effects only for the variables of interest and not for covariates included in the models (e.g. task order, trial repetition); the findings pertaining to the covariates are reported in the Supplement. For the mixed effects logistic regressions modeling choice of the riskier option on the VGT (see below), we report odds ratios as a measure of effect size for the statistically significant effects only. Because sign-tracking propensity followed a gaussian rather than a bimodal distribution in both studies (see below, Figure 2A, 3A), we used the degree of sign-tracking / goal-tracking as a dimensional variable in our primary analyses. We report the analyses defining STs and GTs categorically (using a tertiary split) in the Supplement.

\section{Pavlovian task}

We quantified sign tracking and goal tracking as the proportion of time spent gazing at the reward-predictive CS+ (the sign) versus the location of the impending reward delivery (the goal) during the CS presentation. Because early gaze responses to CS presentation may reflect orienting responses driven by visual salience rather than CS learning (Gottlieb, 2012)(Yager, Pitchers, Flagel, \& Robinson, 2015), we disregarded the first second of the CS period and focused on the last 4 seconds for the purposes of ST and GT identification, as was done previously (Schad et al., 2020). Two rectangular interest areas (IAs) of equal size were defined around the sign and goal locations; the size of these IAs was determined based on visual inspection of the aggregate fixation duration heatmap (Supplementary Figure S1). The eye tracker's proprietary data analysis software, Data Viewer, was used to extract the measure of "\% 
Risk-promoting effects of cues and sign-tracking

dwell time" - the proportion of time during the last 4 seconds of the CS phase spent fixating on each IA. We excluded participants whose eye data were missing in $>50 \%$ of the trials (5 participants) and those that spent $>80 \%$ of this interest period gazing at neither the CS or the US (5 participants), which we assumed to reflect a lack of engagement in the task. In addition, Pavlovian task data were lost for one participant because of equipment failure. Of the 131 participants tested, 120 were entered into the subsequent analyses. We then calculated a "gaze index" for each participant as the difference between the average \% dwell time for the sign and goal IAs (\% dwell time (sign) - \% dwell time (goal)). The index ranged from -1 to 1, with higher values corresponding to more sign-tracking. We computed this gaze index separately for the first 20 trials (hemiblock 1) and the last 20 trials (hemiblock 2). We took gaze index in hemiblock 2 as the measure of sign-tracking CR, as was done previously (Garofalo \& di Pellegrino, 2015), assuming that the first 20 trials were sufficient for the CR to develop.

To determine whether the Pavlovian task resulted in conditioning, we examined subjective and pupillary measures of Pavlovian conditioning. Linear regression was used to model self-reported liking of the CS stimuli as a function of CS type (CS+ vs. CS-) in interaction with gaze index and task version as a covariate. Because the pupil is known to dilate during learned anticipation of rewards and in response to uncertainty (Pietrock et al., 2019)(Nassar et al., 2012), and pupillary responses to reward anticipation during CS presentation have been reported to differ between ST and GT (Schad et al., 2020), we examined pupil responses to CS+ vs CS- as a function of phenotype. Pupil area time-series were extracted for each participant and processed using MATLAB scripts. First, linear regression was used to correct pupil area measures for vertical and horizontal gaze position, as pupil foreshortening at eccentric gaze positions can affect pupil size measurements (Hayes \& Petrov, 2016). All subsequent analyses 
Risk-promoting effects of cues and sign-tracking

were performed on the standardized residuals. Outlier values $>$ or $<2 \mathrm{SD}$ of the mean were removed. Linear interpolation was performed over all samples occurring during blinks and outliers removed. The pupil time series were then smoothed using a second-order Butterworth low pass filter with the cut-off frequency of $4 \mathrm{~Hz}$. Conditioned pupil response was quantified as area under the curve (AUC) over the duration of CS presentation with respect to baseline, which was defined as the pupil size at 500ms following CS onset to account for the lag in pupil response (Clayton, 2004)(Partala \& Surakka, 2003). These AUC values were analyzed using a linear mixed effects model predicting pupil dilation from gaze index in interaction with CS type and trial number in the task, with random intercepts modelled for participants and random slopes for hemiblock and CS, and task version as a covariate.

$$
\text { AUC(pupil) gaze index* CS*trial + version }+(1+\mathrm{CS}+\text { trial } \mid \text { participant })
$$

\section{Decision making}

The effect of cues and phenotype on VGT performance was analyzed using linear mixed effects models with a logistic link (glmer function). We modelled the likelihood of choosing the lower-probability (riskier) prospect versus a higher-probability (safer) prospect on a trial-by-trial basis as a function of gaze index in interaction with cues and EVR. Additional fixed effects were modeled for task order (VGT first vs. VGT second) and for trial repetition, because participants made riskier choices with successive trial repetitions (Cherkasova et al., 2018). The trial repetition variable was centered in all analyses. Random intercepts were modelled for participants and random slopes for EVR and trial repetition and EVR.

$\mathrm{p}($ risky $) \sim$ gaze index $*$ cues $*$ EVR + task_order + repetition $+($ EVR + repetition $\mid$ participant $)$ 
Risk-promoting effects of cues and sign-tracking

\section{Results}

Sign-Tracking and Goal-Tracking

Distribution of the gaze index in the second hemiblock (Figure 2A) was skewed towards sign-tracking with the median gaze index of 0.27 .

\section{Pavlovian Conditioning}

Participants developed a significant subjective preference for the CS+ relative to the CS$(b=1.62, S E=0.17, t=9.82, p<0.0005$, Figure $2 B)$, and there was no significant interaction with gaze index $(\mathrm{p}=0.75)$.

Participants displayed a pupil dilation response during the CS presentation, ramping up as the time of reward delivery approached (Figure 2C). There was a significant decrease in the magnitude of this response as a function of trial number $(b=-0.30, \mathrm{SE}=0.03, \mathrm{t}=-6.76, \mathrm{p}<$ 0.0005), consistent with a reduction in uncertainty-driven pupil response as the CS-US association is learned. There was also a significant CS $\mathrm{x}$ trial interaction with a greater reduction in the anticipatory pupil dilation for the CS- than the $\mathrm{CS}+(\mathrm{b}=0.08, \mathrm{SE}=0.03, \mathrm{t}=2.61, \mathrm{p}=0.009)$. The smaller reduction in pupil response to the CS+ could reflect greater reward anticipation when presented with the CS+ on top of uncertainty reduction. Alternatively, it could reflect less uncertainty reduction for the probabilistic CS+. There were no significant interactions with gaze index $(\mathrm{p} \leq 0.29)$ suggesting that these effects did not differ as a function of sign-tracking propensity.

\section{Decision Making}

As reported previously (Cherkasova et al., 2018), there was a significant effect of cues on the propensity to choose riskier prospects (associated with lower reward probabilities) on the 
Risk-promoting effects of cues and sign-tracking

Vancouver Gambling Task $(b=-0.76, \mathrm{SE}=0.21, \mathrm{t}=3.57, \mathrm{OR}[95 \% \mathrm{CI}]=0.47[0.26,0.91], \mathrm{p}=$ 0.003). There also was a significant gaze index $x$ cues interaction such that participants with lower (more GT-like) sign-tracking scores were more likely to take risks in the presence of cues than in their absence $(b=0.90, \mathrm{SE}=0.41, \mathrm{t}=2.19, \mathrm{OR}[95 \% \mathrm{CI}]=2.46[0.69,4.09], \mathrm{p}=0.03)$

(Figure 2D). This was a value-independent effect, as there was no significant interaction of signtracking propensity and cues with expected value of the prospects $(\mathrm{p}=0.12)$.

\section{Study 2}

The aims of this study were as follows. 1) To replicate the effects of sensory reward cues on decision-making we observed in Study 1 using a within-subject design. 2) To modify the Pavlovian paradigm in order to reduce the skew in the gaze index distribution by increasing the size and salience of the reward. 3) To present the visual cues in the VGT at both reward prediction and outcome. Because sign-tracking is defined as the attribution of incentive salience to reward-predictive cues, we reasoned that ST-like participants in Study 1 may not be as sensitive to the VGT cues because these cues were reward-concurrent and therefore did not predict or signal reward. Instead, they may have played a more contextual role, which could explain why participants with a more GT-like CR were preferentially sensitive to them. We therefore modified the cued VGT to introduce the visual cues in a reward-predictive position at the time of choice in addition to those present at the time of feedback.

\section{Materials and Methods}

\section{Participants}

We tested 58 community volunteers (males: $\mathrm{n}=27$, females: $\mathrm{n}=31$, age $=24.09 \pm 5.85$ ). The sample size was based on the medium effect size of cues on decision making from Study 1. 
Risk-promoting effects of cues and sign-tracking

Participants underwent a Structured Clinical Interview for DSM-5 Axis I disorders (SCID-5)

(First, Williams, Karg, \& Spitzer, 2015) and were excluded for any ongoing Axis I disorders or use of psychotropic medication. Other exclusion criteria were the same as in Study 1.

\section{Procedure}

Each participant performed both the cued and the uncued version of the VGT in a withinsubject design with the order randomized across participants. The PIT task was performed between the cued and uncued task versions.

\section{Tasks}

\section{Vancouver Gambling Task}

The uncued version of the task was identical to that in Study 1. The cued version differed in that the magnitudes of the possible outcomes were represented using images of gold coins (Figure 1B).

\section{Pavlovian Conditioning}

The Pavlovian task (Figure 1D) differed from Study 1 in the following ways. In an attempt to increase the goal-tracking $\mathrm{CR}$, we 1) increased the reward size to $\$ 1 ; 2$ ) introduced a consummatory aspect to the feedback by requiring the participants to interact with the reward by "collecting" it via a mouse click on the coin. This also bears a closer resemblance to the rodent paradigm, where the animals consume the food reward, rather then passively observing it. The Canadian $\$ 1$ coin was presented immediately following the CS period, which lasted 7000ms (because the Response phase was absent in Study2, the CS period was made 200ms longer). Participants were given $2000 \mathrm{~ms}$ in which to perform the mouse click. The failure to do so resulted in not earning the reward. Once the reward was successfully collected, participants were 
shown their running total of earnings accompanied by a cash register sound (500ms). Finally, to distinguish liking of the CS from learning of the reward contingencies, we added a self-report estimate of the reward rate associated with each CS at the end of the task.

\section{Analyses}

To maximize correspondence to Study 1, we calculated the gaze index based on the first 5 of the 7 seconds of the CS phase (1-5 seconds after onset) and excluding the first second as the orienting response. Because the cued and uncued VGT were performed in a within-subjects design, random slopes were modelled for cues in the VGT analyses:

$$
\begin{gathered}
\mathrm{p}(\text { risky }) \sim \text { gaze index } * \text { cues* EVR }+ \text { task_order }+ \text { repetition }+(\text { EVR }+ \text { repetition }+ \text { cues } \mid \\
\text { participant })
\end{gathered}
$$

Otherwise, the analyses were the same as in Study 1.

\section{Results}

Sign-Tracking and Goal-Tracking

Two participants were excluded for $>80 \%$ of the CS phase spent gazing at neither the CS nor the US; 56 participants were entered into the subsequent analyses. Distribution of the gaze index in the second hemiblock of the task is given in Figure 3A; median gaze index was 0.03.

\section{Pavlovian Conditioning}

Participants developed a significant subjective preference for the CS+ relative to the CS$(\mathrm{b}=1.2, \mathrm{SE}=0.2, \mathrm{t}=5.86, \mathrm{p}<0.0005)$, and this did not significantly interact with gaze index $(\mathrm{p}=$ 0.89) (Figure 3B). Participants also rated the CS+ as significantly more likely to predict rewards 
Risk-promoting effects of cues and sign-tracking

than the CS- $(b=9.39, S E=0.58, t=16.13, p<0.0005)$, and this did not significantly interact with gaze index $(\mathrm{p}=0.61)$ (Figure 3C).

As in Study 1, participants displayed a pupil dilation response during the CS presentation, ramping up as the time of reward delivery approached (Figure 3D). There was a significant decrease in the magnitude of this response as a function of trial number $(b=-0.34, S E=0.06, t=-$ $6.14, \mathrm{p}<0.0005)$, which is consistent with a reduction in an uncertainty-driven pupil response as a function of learning the CS-US association. A main effect of gaze index $(b=0.23, S E=0.08$, $\mathrm{t}=2.77, \mathrm{p}=0.008$ ) indicated that more ST-like participants displayed greater anticipatory pupil dilation. There was also a gaze index $\mathrm{x} C \mathrm{C}$ interaction $(\mathrm{b}=0.28, \mathrm{SE}=0.12, \mathrm{t}=2.22, \mathrm{p}=0.03)$. Separate follow-up models for the CS+ and the CS- showed that while there was no difference in pupil response to the CS+ as a function of gaze index $\left(\mathrm{p}_{\mathrm{s}} \geq 0.38\right)$, more GT-like participants showed the least anticipatory pupil dilation to the CS- (main effect of gaze index: $b=0.21$, $\mathrm{SE}=0.08, \mathrm{t}=2.62, \mathrm{p}=0.01)$. This suggests that GT-like participants showed either decreased uncertainty or decreased reward anticipation when presented with the CS-.

\section{Decision Making}

There was a significant main effect of cues $(b=-0.27, \mathrm{SE}=0.11, \mathrm{t}=-2.55, \mathrm{OR}[95 \% \mathrm{CI}]=$ $0.76[0.62,0.94], \mathrm{p}=0.01)$ and a significant cues $\mathrm{x}$ EVR interaction $(\mathrm{b}=-0.32, \mathrm{SE}=0.10, \mathrm{t}=3.08$, $\mathrm{OR}[95 \% \mathrm{CI}]=0.73[0.59,0.89], \mathrm{p}=0.002)$. Cues promoted riskier choice preferentially for prospects with higher expected values. However, gaze index did not interact significantly with cues $(p=0.50)$, and the gaze index $x$ cues $x$ EVR interaction was not significant $(p=0.19)$, although the direction of findings was the same as in Study 1 (Figure 3E), and with the supplementary categorical analysis (Supplement) showing a risk-promoting effect of cues uniquely in GTs. 
Risk-promoting effects of cues and sign-tracking

\section{Discussion}

Here, we examined cost-benefit decision making in human sign-trackers and goaltrackers. Specifically, we asked whether STs and GTs would differ in susceptibility to the riskpromoting effect of sensory reward cues reported earlier by our group (Cherkasova et al., 2018). Contrary to our hypothesis, Study 1 revealed that participants with more GT-like Pavlovianconditioned approach behaviour were more susceptible to the effect of cues. Although we did not replicate this effect in Study 2, the findings were in the same direction, and our secondary analyses with STs and GTs defined categorically using a tertiary split showed an increased riskpromoting effect in GTs relative to STs (see Supplement). Overall, the findings further our understanding of the translational validity of the ST and GT phenotypes in humans and raise the possibility that sensory reward cues - at least the kind tested here - promote risky decision making through other mechanisms than eliciting Pavlovian approach towards larger riskier rewards.

Assuming that sign-tracking, as measured in our study, reflects an enhanced attribution of incentive salience and Pavlovian-conditioned approach to reward cues, we would expect a greater risk-promoting effect of cues in ST-like participants, if it depended on Pavlovian approach. Alternatively, reward cues may be biasing decision making in our paradigm via mechanisms that are distinct and largely independent from Pavlovian-conditioned approach. Although the mechanisms whereby cues promote riskier choice in rodents and humans are not entirely clear, the finding that cue-induced risky choice on the rodent gambling task was unrelated to sign-tracking or conditioned reinforcement supports the possibility of independent mechanisms (Ferland et al., 2019). Furthermore, computational modeling of the rodent data suggests that, on the cued rGT, subjects fail to adequately update the value estimates of different 
Risk-promoting effects of cues and sign-tracking

options sufficiently following losses, whereas the value adjustment following rewarding outcomes does not change significantly as a function of cues (Langdon, Hathaway, Zorowitz, Harris, \& Winstanley, 2019).

This is surprising, in that cues do not occur on loss trials. In our previous study comparing performance on the cued vs uncued VGT, we observed that participants spent less time viewing gamble probability information in the cued task (Cherkasova 2018). If the cues were facilitating value-based attentional capture (Anderson \& Yantis, 2012), we might have expected subjects to spend more time focused on the potential winnings or current reward tally, but this was not the case. Furthermore, pupil dilation following a cued win was greater during the subsequent decision-making phase of the next trial. Findings from another group have pointed to an enhancement of memory for wins as a possible mechanism of the risk-promoting effect of casino-inspired reward cues (Ludvig et al., 2015)(Spetch et al., 2020). Although these casinoinspired reward cues increased approach, this was independent of their risk-promoting effect (Spetch et al., 2020). Collectively, such findings suggest that attention, the general arousal profile, or memory of rewards may be altered by reward cues, and this may affect evaluative processes not directly related to reward amplification.

An alternative explanation for our unexpected findings is that our measure of signtracking does not entirely capture the construct measured by the animal models. Self-reports of CS liking and reward predictiveness together with pupil dilation data during Pavlovian conditioning suggest that the task produced the intended CS-reward associations. However, eyegaze dwelling that was used to quantify sign-tracking could still be questioned as an index of Pavlovian-conditioned approach, despite the evidence of Pavlovian learning. Indeed, in Study 2, pupillometry showed that GT-like participants reduced their anticipatory pupil response to the 
Risk-promoting effects of cues and sign-tracking

CS- more than the CS+, suggesting enhanced stimulus discrimination. This could indicate either that participants with a tendency to goal-track are better learners or that they attribute more incentive salience to the CS+. The former seems less likely given the absence of significant differences in ratings of CS liking or reward predictiveness as a function of gaze index, although pupil dilation may be a more sensitive measure of Pavlovian learning than self-report scales. If the GT-like participants indeed attribute more incentive salience to reward cues, the cues could have promoted riskier choice through Pavlovian approach to riskier rewards in these participants.

Notably, these pupillometry findings are at odds with (Schad et al., 2020) who used a similar eye tracking based method of identifying STs and GTs but reported a reduction in uncertainty-driven pupil dilation uniquely in GTs and the emergence of reward-driven responses uniquely in STs. Together with neuroimaging evidence and computational modeling, this pattern of findings was interpreted to suggest that model-based learning mechanisms dominate in GTs, whereas model-free mechanisms dominate in STs. Notably, the Pavlovian paradigm in (Schad et al., 2020) involved both appetitive and aversive conditioning, which might have affected phenotype classification. Although most extant studies have used paradigms similar to ours relying on eye tracking to measure Pavlovian-conditioned approach (Garofalo \& di Pellegrino, 2015)(Schad et al., 2020)(Doran, 2016), other operationalizations of sign-tracking have also been attempted (Joyner et al., 2018)(Versace, Kypriotakis, Basen-Engquist, \& Schembre, 2016), and it is too early to tell which ones have most translational validity.

In Study 2, we did not replicate the effect of gaze index on the risk-promoting effect of cues in Study 1, although the direction of the findings was consistent with this effect, and our secondary analyses with STs and GTs defined categorically using a tertiary split showed an increased risk-promoting effect in GTs relative to STs (see Supplement). Because the Pavlovian 
paradigm was modified in Study 2 to yield more goal-tracking, the two studies might have had slightly different sign-tracking CR and phenotype definitions, which could have in turn affected the modulation of cue-induced risky choice by sign-tracking. Notably, the risk-promoting effect of cues in Study 2 was value-dependent (cues x EVR interaction), likely owing to the inclusion of the visual cues in the decision phase of the VGT, and the study was not adequately powered to detect a 3-way interaction (gaze index $x$ cues $x$ EVR). In addition, we used the maximally complex random effects structure in our models, which is the most conservative approach. However, our failure to replicate a significant effect of sign-tracking on cue-induced risky choice suggests that this effect may be modest at least in community volunteers not suffering from addictions. The modulatory effect of sign-tracking on cue-induced risky choice (or other forms of cue-reactivity) may be more pronounced in those with addictions, which would imply that ST confers addiction vulnerability by interacting with other addiction-relevant mechanisms or individual characteristics.

As one possible explanation for the unexpected direction of our findings, we considered the role the reward cues played in the context of the VGT. Rather than clearly predicting or incentivising rewards, cues in Study 1 accompanied reward delivery and may have thereby played a more contextual role. This could explain why GTs who have been reported to be preferentially sensitive to the effects of contextual cues were more sensitive to the cue manipulation in Study 1. We attempted to address this possibility in Study 2 by also introducing the visual cues at the time of choice - a position within the task where these cues could have served as reward-predictive incentive signals. This modification resulted in the risk-promoting effect becoming value-dependent, suggesting that incentive-motivational properties of the cues presented at the time of choice indeed translated into increased risk taking selectively for larger 
rewards. Although the findings of Study 2 are inconclusive, their direction is consistent with the findings of Study 1 suggesting that this manipulation did not reverse the effect of sign-tracking on cue-induced risky choice.

In conclusion, we provide the first evidence in humans that goal-trackers are preferentially susceptible to the effects of reward cues in a decision-making context. Signtracking is a promising translational model of addiction, which establishes cue-reactivity as a vulnerability trait and draws important conceptual links with the cue-reactivity states, which are long-studied and well-established predictors of addiction course and outcome in humans (Childress et al., 1993). Our data contribute to the translational validation of the signtracking/goal-tracking propensity as an addiction vulnerability trait. Because decision making is an important candidate mechanism whereby exposure to addiction-related cues may translate into harmful outcomes such as relapse, our findings have implications for understanding the role of sign-tracking in gating the cues' ability to elicit maladaptive choices, such as drug use and pursuit of other addictive behaviours. However, our findings run contrary to the notion that signtrackers are preferentially vulnerable to the influence of reward cues and instead suggest that goal-trackers may be more susceptible to their influence in the decision-making context. As such, these findings raise questions about the translational validity and significance of the signtracker and goal-tracker phenotypes in humans as currently conceptualised and highlight the need for further study in this area. 
Risk-promoting effects of cues and sign-tracking

\section{ACKNOWLEDGMENTS:}

Funding: This work was supported by National Center for Responsible Gaming (NCRG) seed funding awarded to MVC and research allowance funds from post-doctoral fellowships from CIHR and the Michael Smith Foundation for Health Research by which MVC was supported. This work was also partially supported by an operating grant awarded to CAW, LC and AJS from the Canadian Institutes for Health Research (CIHR; PJT-056012). Mariya Cherkasova has received a speaker's honorarium from the Responsible Gaming Association of New Mexico. In the past three years, CAW has consulted for Shire and Hogan Lovells LLP and received due compensation. Eve H. Limbrick Oldfield worked as a postdoctoral fellow at the Centre for Gambling Research at UBC, and Luke Clark is the Director of the Centre for Gambling Research at UBC, which is supported by funding from the Province of British Columbia and the British Columbia Lottery Corporation (BCLC), a Canadian Crown Corporation. The Province of British Columbia government and BCLC had no involvement in the ideas expressed herein, and impose no constraints on publishing. ELO has received a speaker honorarium from the Massachusetts Council on Compulsive Gambling (U.S.A.) and accepted travel/accommodation for speaking engagements from the National Council for Responsible Gambling (U.S.A.), the International Multidisciplinary Symposium on Gambling Addiction (Switzerland) and the Responsible Gambling Council (Canada). She has not received any further direct or indirect payments from the gambling industry or groups substantially funded by gambling. LC receives funding from the Natural Sciences and Engineering Research Council (Canada). LC has received a speaker/travel honorarium from the National Association for Gambling Studies (Australia) and the National Center for Responsible Gaming (US), and has received fees for academic services from the National Center for Responsible Gaming (US), GambleAware (UK), and Gambling Research Exchange Ontario (Canada). He has not received any further direct or indirect payments from the gambling industry or groups substantially funded by gambling. He has provided paid consultancy to, and received royalties from, Cambridge Cognition Ltd. relating to neurocognitive testing. AJS is supported by Canada Research Chairs and has research support from Michael J Fox Foundation and Weston Brain Institute. He is the Editor-in-Chief of Movement Disorders (stipend) and has received compensation as Chair of the Data Safety Monitoring Board for a trial of gene therapy in Parkinson's Disease (Voyager/Neurocrine) and as an advisor for SioGene. The authors confirm they have no other conflicts of interest or financial disclosures to make. 
Risk-promoting effects of cues and sign-tracking

Open Practices Statement:

Data and analysis scripts are available on https://osf.io/9b34v/ and

https://github.com/mcherkasova/STGT_VGT. None of the experiments was preregistered.

\section{REFERENCES}

Adams, W. K., Barkus, C., Ferland, J. M. N., Sharp, T., \& Winstanley, C. A. (2017).

Pharmacological evidence that 5-HT2C receptor blockade selectively improves decision making when rewards are paired with audiovisual cues in a rat gambling task. Psychopharmacology, 234(20), 3091-3104. https://doi.org/10.1007/s00213-017-4696-4

Anderson, B. A., \& Yantis, S. (2012). Value-driven attentional and oculomotor capture during goal-directed, unconstrained viewing. Attention, Perception, \& Psychophysics, 74(8), 16441653. https://doi.org/10.3758/s13414-012-0348-2

Barrus, M. M., \& Winstanley, C. A. (2016). Dopamine D3 Receptors Modulate the Ability of Win-Paired Cues to Increase Risky Choice in a Rat Gambling Task. Journal of Neuroscience, 36(3), 785-794. https://doi.org/10.1523/JNEUROSCI.2225-15.2016

Bates, D., Mächler, M., Bolker, B., \& Walker, S. (2015). Fitting Linear Mixed-Effects Models using lme4. Journal of Statistical Software, 67(1), 1-48. https://doi.org/10.18637/jss.v067.i01

Boswell, R. G., Kober, H., Haven, N., \& Haven, N. (2018). HHS Public Access. Obesity Reviews, 17(2), 159-177. https://doi.org/10.1111/obr.12354.Food

Carter, B. L., \& Tiffany, S. E. N. T. (1999). Meta-analysis of cue-reactivity in addiction research. Analysis, 94(April 1998), 327-340.

Cherkasova, M. V, Clark, L., Barton, J. J. S., Schulzer, M., Shafiee, M., Kingstone, A., ... Winstanley, C. A. (2018). Win-Concurrent Sensory Cues Can Promote Riskier Choice. Journal of Neuroscience, 38(48), 10362-10370.

Childress, A. R., Hole, A. V., Ehrman, R. N., Robbins, S. J., McLellan, T. E., \& O’Brien, C. P. (1993). Clinicwide and individualized behavioral interventions in drug dependence treatment. NIDA Res Monogr, 137(April 2014), 37-72. 
Risk-promoting effects of cues and sign-tracking

https://doi.org/10.1016/j.matcom.2015.04.007

Clayton, E. C. (2004). Phasic Activation of Monkey Locus Ceruleus Neurons by Simple

Decisions in a Forced-Choice Task. Journal of Neuroscience, 24(44), 9914-9920. https://doi.org/10.1523/JNEUROSCI.2446-04.2004

Doran, K. S. (2016). Translational approaches to studying reward-based purposive behaviours.

Ferland, J. M. N., Hynes, T. J., Hounjet, C. D., Lindenbach, D., Vonder Haar, C., Adams, W. K., ... Winstanley, C. A. (2019). Prior exposure to salient win-paired cues in a rat gambling task increases sensitivity to cocaine self-administration and suppresses dopamine efflux in nucleus accumbens: Support for the reward deficiency hypothesis of addiction. Journal of Neuroscience, 39(10), 1842-1854. https://doi.org/10.1523/JNEUROSCI.3477-17.2018

Field, M., \& Cox, W. M. (2008). Attentional bias in addictive behaviors: A review of its development, causes, and consequences. Drug and Alcohol Dependence, 97(1-2), 1-20. https://doi.org/10.1016/j.drugalcdep.2008.03.030

First, M. B., Spitzer, R. L., Gibbon, M., \& Williams, J. B. W. (1999). Structured Clinical interview for DSM-IV Axis I disorders. New York: Biometrics Research Department, New York State Psychiatric Institute. https://doi.org/10.1093/molbev/msp224

First, M. B., Williams, J. B. W., Karg, R. S., \& Spitzer, R. L. (2015). Structured Clinical Interview for DSM-5 -- Research Version (SCID-5 for DSM-5, Research Version; SCID-5$R V)$. American Psychiatric Association.

Flagel, S. B., Akil, H., \& Robinson, T. E. (2009). Individual differences in the attribution of incentive salience to reward-related cues: Implications for addiction. Neuropharmacology, 56(SUPPL. 1), 139-148. https://doi.org/10.1016/j.neuropharm.2008.06.027

Garofalo, S., \& di Pellegrino, G. (2015). Individual differences in the influence of task-irrelevant Pavlovian cues on human behavior. Frontiers in Behavioral Neuroscience, 9(June), 163. https://doi.org/10.3389/fnbeh.2015.00163

Gottlieb, J. (2012). Attention, learning, and the value of information. Neuron, 76(2), 281-295. https://doi.org/10.1016/j.neuron.2012.09.034 
Goudriaan, A. E., De Ruiter, M. B., Van Den Brink, W., Oosterlaan, J., \& Veltman, D. J. (2010). Brain activation patterns associated with cue reactivity and craving in abstinent problem gamblers, heavy smokers and healthy controls: An fMRI study. Addiction Biology, 15(4), 491-503. https://doi.org/10.1111/j.1369-1600.2010.00242.x

Hayes, T. R., \& Petrov, A. A. (2016). Mapping and correcting the influence of gaze position on pupil size measurements. Behavior Research Methods, 48(2), 510-527. https://doi.org/10.3758/s13428-015-0588-x

Jasinska, A. J., Stein, E. A., Kaiser, J., Naumer, M. J., \& Yalachkov, Y. (2014). Factors modulating neural reactivity to drug cues in addiction: a survey of human neuroimaging studies. Neuroscience \& Biobehavioral Reviews, 38, 1-16. https://doi.org/10.1016/j.neubiorev.2013.10.013

Joyner, M. A., Gearhardt, A. N., \& Flagel, S. B. (2018). A Translational Model to Assess SignTracking and Goal-Tracking Behavior in Children. Neuropsychopharmacology, 43(1), 228229. https://doi.org/10.1038/npp.2017.196

Langdon, A. J., Hathaway, B. A., Zorowitz, S., Harris, C. B. W., \& Winstanley, C. A. (2019). Relative insensitivity to time-out punishments induced by win-paired cues in a rat gambling task. Psychopharmacology, 236(8), 2543-2556. https://doi.org/10.1007/s00213-019-05308$\mathrm{x}$

Limbrick-Oldfield, E. H., Mick, I., Cocks, R. E., McGonigle, J., Sharman, S. P., Goldstone, A. P., ... Clark, L. (2017). Neural substrates of cue reactivity and craving in gambling disorder. Translational Psychiatry, 7(1), e992. https://doi.org/10.1038/tp.2016.256

Ludvig, E. A., Madan, C. R., \& Spetch, M. L. (2015). Priming memories of past wins induces risk seeking. Journal of Experimental Psychology: General, 144(1), 24-29. https://doi.org/10.1037/xge0000046

MacNiven, K. H., Jensen, E. L. S., Borg, N., Padula, C. B., Humphreys, K., \& Knutson, B. (2019). Association of Neural Responses to Drug Cues With Subsequent Relapse to Stimulant Use. JAMA, 1(8), 1-14. https://doi.org/10.1001/jamanetworkopen.2018.6466

Meyer, P. J., Lovic, V., Saunders, B. T., Yager, L. M., Flagel, S. B., Morrow, J. D., \& Robinson, 
Risk-promoting effects of cues and sign-tracking

T. E. (2012). Quantifying individual variation in the propensity to attribute incentive salience to reward cues. PLoS ONE, 7(6). https://doi.org/10.1371/journal.pone.0038987

Nassar, M. R., Rumsey, K. M., Wilson, R. C., Parikh, K., Heasly, B., \& Gold, J. I. (2012). Rational regulation of learning dynamics by pupil-linked arousal systems. Nature Neuroscience, 15(7), 1040-1046. https://doi.org/10.1038/nn.3130

Nieuwenhuis, R., te Grotenhuis, M., \& Pelzer, B. (2012). Influence.ME: Tools for detecting influential data in mixed effects models. $R$ Journal, 4(2), 38-47. https://doi.org/10.32614/rj2012-011

Norberg, M. M., Kavanagh, D. J., Olivier, J., \& Lyras, S. (2016). Craving cannabis: a metaanalysis of self-report and psychophysiological cue-reactivity studies. Addiction (Abingdon, England), 111(11), 1923-1934. https://doi.org/10.1111/add.13472

Partala, T., \& Surakka, V. (2003). Pupil size variation as an indication of affective processing. International Journal of Human Computer Studies, 59(1-2), 185-198. https://doi.org/10.1016/S1071-5819(03)00017-X

Pietrock, C., Ebrahimi, C., Katthagen, T. M., Koch, S. P., Heinz, A., Rothkirch, M., \& Schlagenhauf, F. (2019). Pupil dilation as an implicit measure of appetitive Pavlovian learning. Psychophysiology, 56(12), 1-18. https://doi.org/10.1111/psyp.13463

Robinson, T. E., \& Berridge, K. C. (1993). The neural basis of drug craving: An incentivesalience theory of addiction. Brain Research Reviews, 18, 247-291. https://doi.org/10.1016/0165-0173(93)90013-P

Robinson, T. E., Yager, L. M., Cogan, E. S., \& Saunders, B. T. (2014). On the motivational properties of reward cues: Individual differences. Neuropharmacology, 76(PART B), 450459. https://doi.org/10.1016/j.neuropharm.2013.05.040

Saunders, B. T., O’Donnell, E. G., Aurbach, E. L., \& Robinson, T. E. (2014). A cocaine context renews drug seeking preferentially in a subset of individuals. Neuropsychopharmacology, 39(12), 2816-2823. https://doi.org/10.1038/npp.2014.131

Saunders, B. T., \& Robinson, T. E. (2010). A Cocaine Cue Acts as an Incentive Stimulus in Some but not Others: Implications for Addiction. Biological Psychiatry, 67(8), 730-736. 
https://doi.org/10.1016/j.biopsych.2009.11.015

Saunders, B. T., \& Robinson, T. E. (2011). Individual Variation in the Motivational Properties of Cocaine. Neuropsychopharmacology, 36(8), 1668-1676.

https://doi.org/10.1038/npp.2011.48

Saunders, B. T., \& Robinson, T. E. (2013). The role of dopamine in the expression of Pavlovian conditioned responses. European Journal of Neuroscience, 36(4), 2521-2532. https://doi.org/10.1111/j.1460-9568.2012.08217.x.The

Schad, D. J., Rapp, M. A., Garbusow, M., Nebe, S., Sebold, M., Obst, E., ... Huys, Q. J. M. (2020). Dissociating neural learning signals in human sign- and goal-trackers. Nature Human Behaviour, 4(2), 201-214. https://doi.org/10.1038/s41562-019-0765-5

Spetch, M. L., Madan, C. R., Liu, Y. S., \& Ludvig, E. A. (2020). Effects of winning cues and relative payout on choice between simulated slot machines. Addiction, 115(9), 1719-1727. https://doi.org/10.1111/add.15010

Versace, F., Kypriotakis, G., Basen-Engquist, K., \& Schembre, S. M. (2016). Heterogeneity in brain reactivity to pleasant and food cues: evidence of sign-tracking in humans. Social Cognitive and Affective Neuroscience, 11(4), 604-611. https://doi.org/10.1093/scan/nsv143

Wardle, M. C., Lopez-Gamundi, P., \& Flagel, S. B. (2018). Measuring appetitive conditioned responses in humans. Physiology and Behavior, 188(February), 140-150. https://doi.org/10.1016/j.physbeh.2018.02.004

Wynne, H. (2003). Introducing the Canadian problem gambling index. Edmonton, AB: Wynne Resources.

Yager, L. M., Pitchers, K. K., Flagel, S. B., \& Robinson, T. E. (2015). Individual variation in the motivational and neurobiological effects of an opioid cue. Neuropsychopharmacology, 40(5), 1269-1277. https://doi.org/10.1038/npp.2014.314

Yager, L. M., \& Robinson, T. E. (2013). A classically conditioned cocaine cue acquires greater control over motivated behavior in rats prone to attribute incentive salience to a food cue. Psychopharmacology (Berlin)), 226(2), 217-228. https://doi.org/10.1007/s00213-012-2890y.A 
Risk-promoting effects of cues and sign-tracking

\section{Figure legends}

\section{Figure 1: Tasks}

A) Study 1, cued and uncued Vancouver Gambling Task: gain magnitudes represented using numerals beneath the pie charts, indicating the number of tokens that could be won $(1,2,3,4$ or 5), with each token worth $\notin 10$ Canadian in actual money. B) Study 2, cued and uncued Vancouver Gambling Task: gain magnitudes represented using gold coins beneath the pie charts to indicate the number of tokens that could be won. Pie charts represent probabilities for each option: $20 \%$ vs. $80 \%, 30 \%$ vs. $70 \%$, or $40 \%$ vs. $60 \%$ in both studies. The decision phase of the task was followed by an anticipation phase with a spinning roulette display, and a feedback phase with (cued) or without (uncued) the audiovisual cues; these phases were identical across Studies 1 and 2. C) Study 1, Pavlovian paradigm. D) Study 2, Pavlovian paradigm. ITI = intertrial interval; $\mathrm{CS}=$ conditioned stimulus.

Figure 2: Study 1, Pavlovian Conditioning and Decision Making

A) Gaze index distribution. B) Subjective liking ratings for CS+ and CS-. C) Pupil dilation responses to CS+ and CS-. For illustration purposes, pupil dilation plots are shown in STs and GTs defined categorically as groups using tertiary split, see Supplement. Error ribbons represent standard error of the mean. D) The effect of cues on decision making as a function of signtracking (defined dimensionally). The lines represent predicted probabilities of risky choice; ribbons represent standard error of the mean.

The three panels depict the effect of cues on decision making at three levels of sign-tracking propensity: gaze index $=-0.15$, goal-tracking; gaze index $=0.29$, intermediate; gaze index $=$ 0.72 , sign-tracking. $* * * \mathrm{p}<0.0005$.

\section{Figure 3: Study 1, Pavlovian Conditioning and Decision Making}

A) Gaze index distribution. B) Subjective liking ratings for CS+ and CS-. C) Reward likelihood ratings for CS+ and CS-. D) Pupil dilation responses to CS+ and CS-. Error ribbons represent standard error of the mean. E) The effect of cues on decision making as a function of signtracking (defined dimensionally). The lines represent predicted probabilities of risky choice; ribbons represent standard error of the mean. The three panels depict the effect of cues on decision making at three levels of sign-tracking propensity: gaze index $=-0.15$, goal-tracking; gaze index $=0.29$, intermediate; gaze index $=0.72$, sign-tracking. $* * * p<0.0005$. 
Figure 1: Tasks

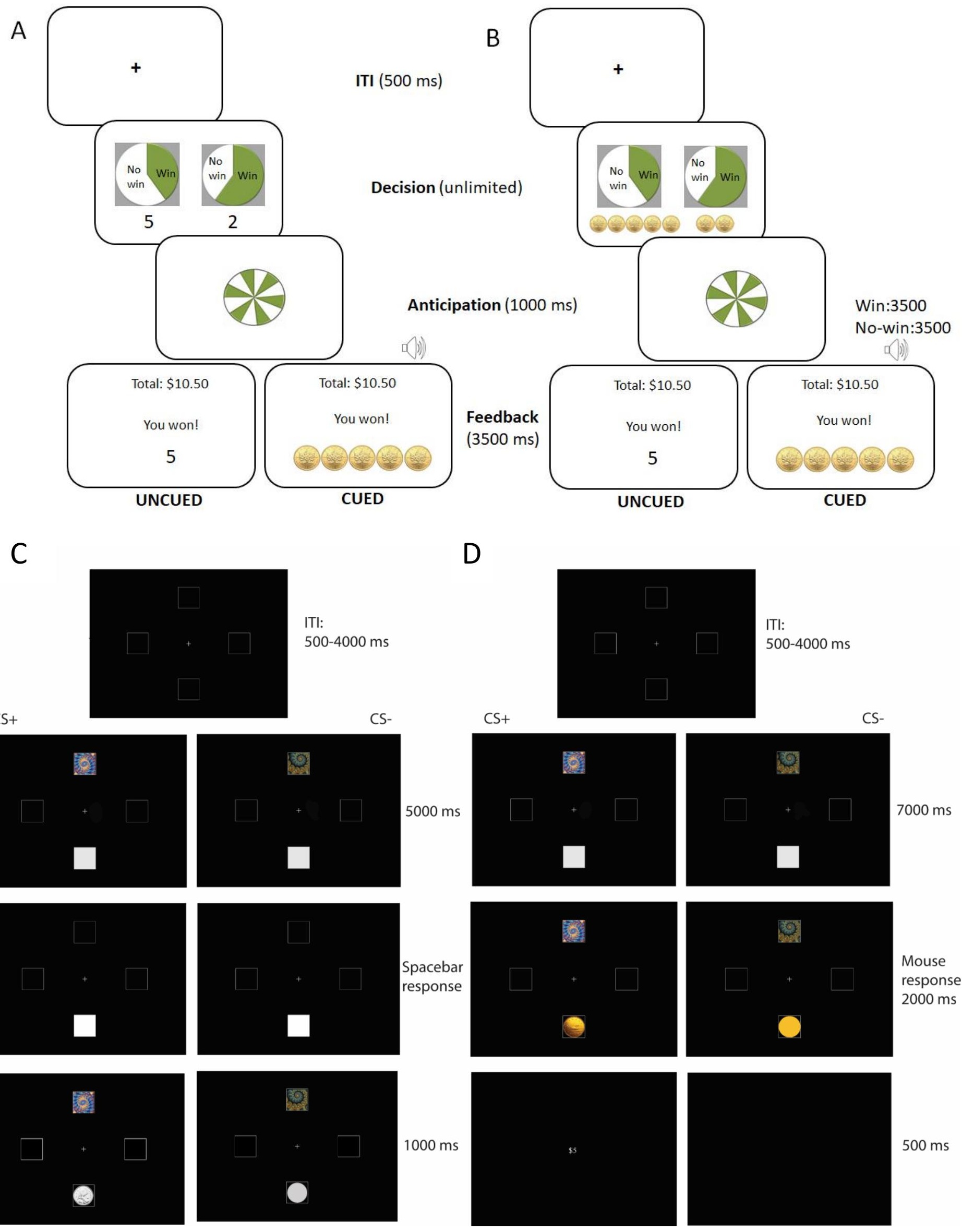


Figure 2
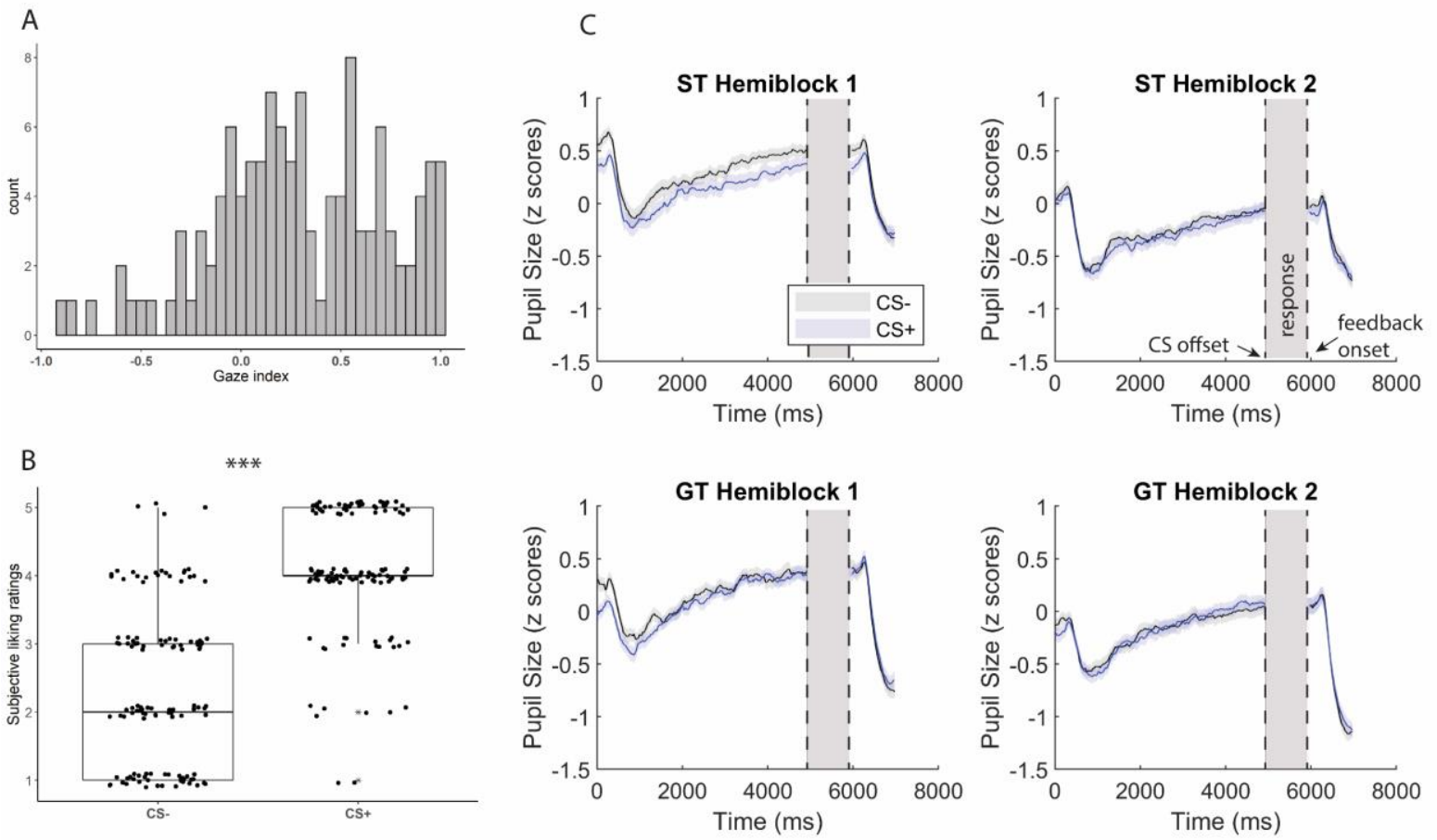

D
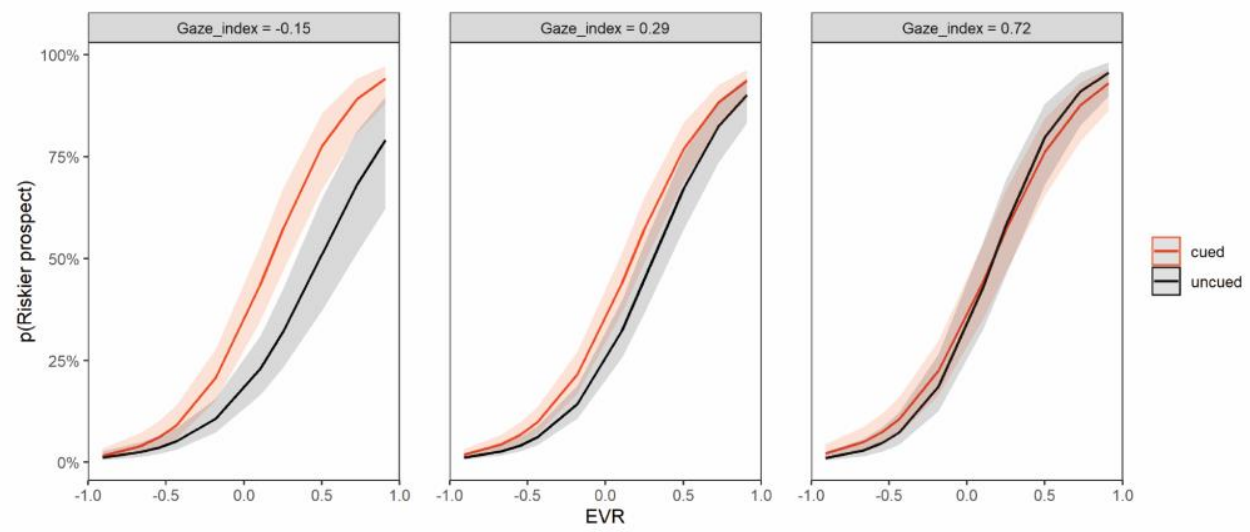
Figure 3

A

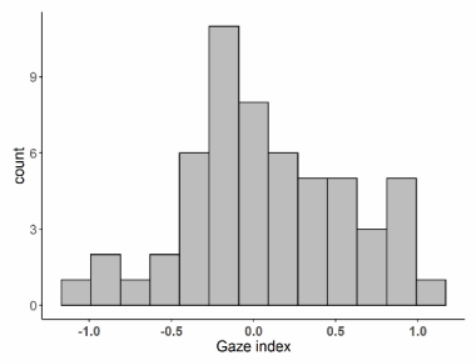

D
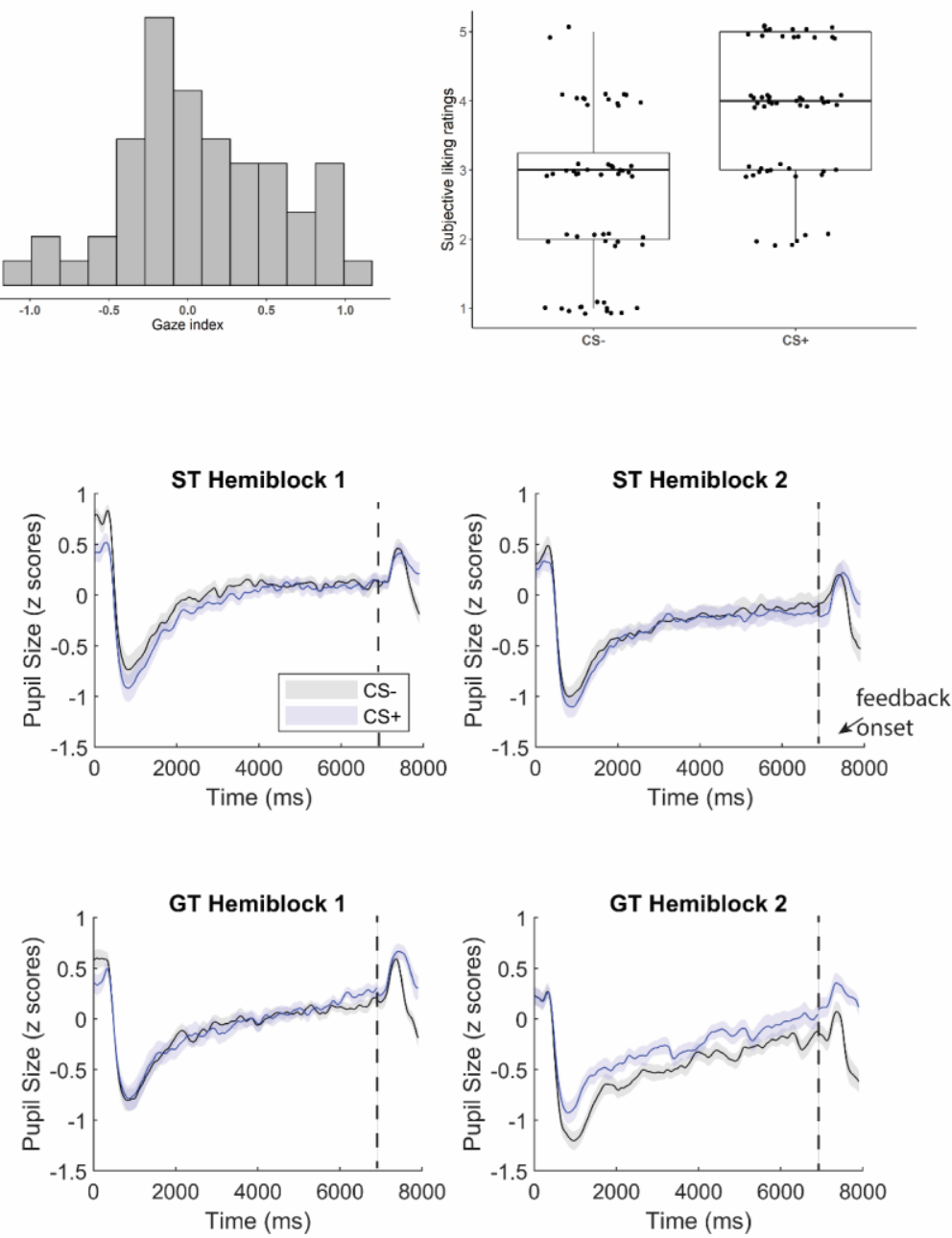

E
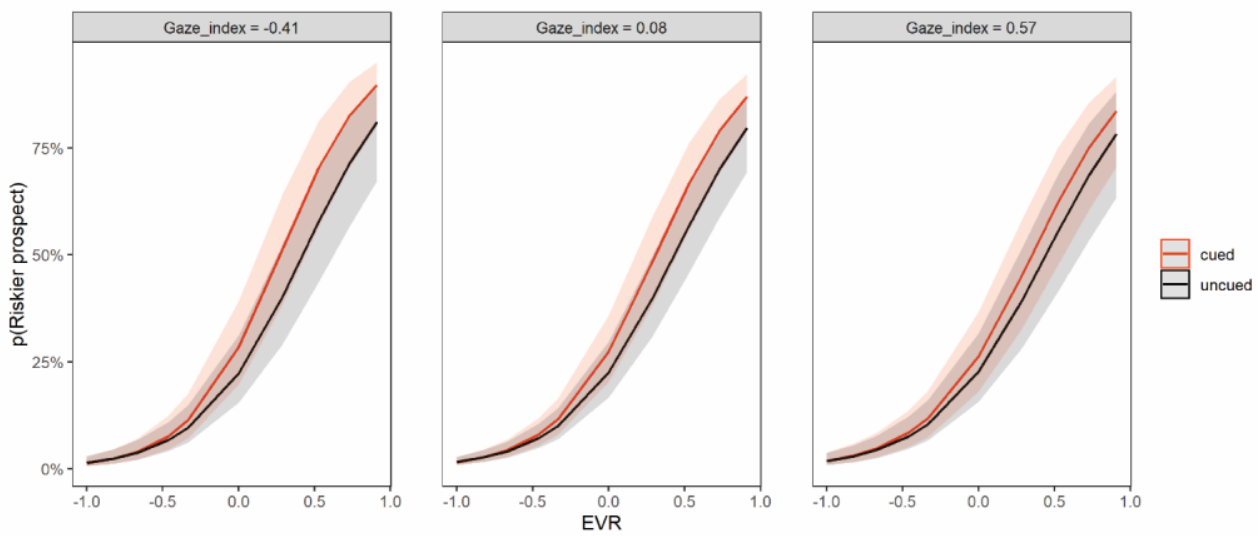

C ***

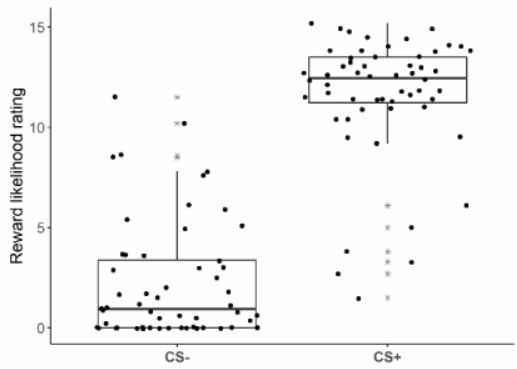




\section{Supplementary Methods}

\section{Eye Tracking}

Most participants (Study 1: $\mathrm{n}=85$; Study 2: $\mathrm{n}=54$ ) were tested on the EyeLink 1000 Tower system. The remaining participants were tested using the EyeLink 1000 Desktop system, either because they required glasses or because the Tower system was unavailable. Participants were tested in one of two labs, in which the apparatus differed slightly. The majority of participants $(n=115)$ were tested in a slightly dimmed lab (illumination $=80$ lux $)$ with the eyes positioned at the distance of $60 \mathrm{~cm}$ from a 22 ' monitor. Sixteen participants in Study 1 were tested using the Desktop system in a lab without a dimmer, so the testing room was dark (1 lux); the monitor was $17^{\prime}$ ' in size, so the viewing distance (normally $57 \mathrm{~cm}$ ) was adjusted accordingly $(47 \mathrm{~cm})$ to preserve stimulus size in visual angle. In study 2 , all participants were tested in the lab with slightly dimmed illumination (80 lux) with the eyes positioned at the distance of $60 \mathrm{~cm}$ from a 22 " monitor.

\section{Pavlovian Instrumental Transfer}

The same visual background was used in all 3 tasks (Instrumental, Pavlovian, PIT): four squares $\left(4 \mathrm{~cm}^{2}\right)$ displayed on a black background and positioned at top center, bottom center, left center and right center of the screen.

\section{Instrumental conditioning}

Task: For Study 1, in the instrumental conditioning phase (Figure S1), the lateral squares were white. Participants were instructed that they could win coins by clicking on these squares with the mouse, but that they would only win rewards some of the time. They were instructed to maximize their rewards and were told that they were free to perform as many clicks as they 
wished. Both squares yielded rewards on a variable interval schedule (every 4-12 seconds), but one of them always yielded a larger reward ( $25 \not$ vs. $5 \not)$. Rewards were presented in the bottom center square using images of the $25 \notin$ and $5 \notin$ Canadian coins; non-rewarded outcomes were represented using a silver circle of the luminance and hue corresponding to that of the coins. The reward or non-reward feedback was presented immediately after every click inside one of the lateral squares for $500 \mathrm{~ms}$. The location of the larger reward was counterbalanced across participants. The goal of the task was for the participants to learn the association between a specific response and the larger reward. The task lasted $\sim 6$ minutes, during which participants could perform as many responses as they wanted. It was accompanied by eye tracking, but the eye tracking data were not analyzed. Instrumental conditioning was measured as the proportion of clicks on the more rewarded location. The instrumental task in Study 2 was the same as in Study 1, except that only one of the lateral squares was associated with a reward (25ф). This simplification was made with the goal of increasing the success or instrumental learning, which nonetheless remained similar across the two studies (see Results).

Analysis: We quantified instrumental learning as the proportion of responses on the more rewarded lateral square (henceforth, congruent choices). We evaluated the effect of phenotype (ST vs. GT, see below for phenotype definition) on the rate of congruent choices using a linear regression with task version as a covariate. One sample t-test was used to determine whether the proportion of congruent responses was significantly above chance.

\section{Pavlovian Conditioning}

The Pavlovian task for both Studies is described in detail in the main manuscript. 
Analysis: Gaze index calculation is described in detail in the main manuscript. For the categorical analysis, we classified participants as ST or GT using a tertiary split on the gaze index in hemiblock 2. Alternative approaches to \% dwell time analysis, such as considering the entire CS phase duration or smaller IAs produced the same pattern of results, as did group definition using a median rather than tertiary split. Analyses of subjective and pupil responses to the conditioned stimuli are described in detail in the manuscript.

\section{Pavlovian Instrumental Transfer}

Task: Participants were informed that this task was similar to the instrumental task, but that obtaining rewards would be harder. The display was the same as for the instrumental task, with two white lateral squares, except that the top square now displayed fractal images whose identity changed throughout the task. These included the CS+ and the CS- fractals, as well as two additional novel fractals to provide a neutral baseline. The CS+ and CS- fractals were each presented 6 times, and the novel fractals were presented once each. Fractals remained on the screen for 30 seconds, during which the participants were free to make as many mouse responses as they wished. As the task was performed in extinction, these mouse responses never yielded rewards. The PIT task was identical across Studies 1 and 2.

Analysis: For PIT analysis, we only considered participants who had successfully learned the operant response, i.e. those whose operant performance was above chance. The threshold for above-chance performance was determined using a permutation approach (1000 random permutations) to determine the proportion of correct responses that can be expected with $95 \%$ confidence as a result of random performance across 445 responses (the mean number of responses generated by participants). Based on this, we excluded participants whose operant performance was $<54 \%$ correct. (Including all participants yielded the same pattern of results.) 
We compared the proportion of congruent choices (those associated with greater reward during instrumental conditioning) vs. incongruent choices during the CS+, the CS- and the neural stimuli. As per (Garofalo \& di Pellegrino, 2015), a response index was calculated as: (number of congruent choices - number of incongruent choices)/(number of congruent choices + number of incongruent choices). A linear regression model was then used to predict response index as a function of fractal stimulus (CS+ vs. CS- and novel stimulus) in interaction with phenotype (ST vs. GT). This was followed by a confirmatory analysis modelling response index as a function of stimulus in interaction with gaze index as a dimensional measure of ST. Similar to (Garofalo \& di Pellegrino, 2015), we performed an additional analysis dividing the task into two equal blocks of three 30-second trials each in the presence of CS+ and CS- and modelling response index as a function of stimulus in interaction with phenotype for each block. This, again, was followed with a confirmatory analysis considering ST as a dimensional variable. Interactions with phenotype were further examined in separate models for ST and GT predicting response index as a function of stimulus.

\section{Decision Making}

Categorical analysis: The effect of cues and phenotype on VGT performance was analyzed using linear mixed effects models with a logistic link (glmer function). We modelled the likelihood of choosing the lower-probability (riskier) prospect versus a higher-probability (safer) prospect on a trial-by-trial basis as a function of phenotype (ST vs. GT) defined categorically using a tertiary split in interaction with cues and EVR. Additional fixed effects were modeled for task order (VGT first vs. VGT second) and for trial repetition, because participants made riskier choices with successive trial repetitions (Cherkasova et al., 2018). The 
trial repetition variable was centered in all analyses. Random intercepts were modelled for participants and random slopes for EVR and trial repetition and EVR.

$$
\text { p(risky) } \sim \mathrm{ST} * \text { cues* EVR + task_order + repetition + (EVR + repetition | participant })
$$

For Study 2 analyses, because the cued and uncued VGT were performed in a within-subjects design, random slopes were modelled for cues.

$$
\mathrm{p}(\text { risky }) \sim \mathrm{ST} * \text { cues } * \text { EVR + task_order + repetition + }(\mathrm{EVR}+\text { repetition + cues } \mid \text { participant })
$$

\section{Supplementary Results}

\section{Study 1}

Sign-Tracking and Goal-Tracking

A tertiary split on the gaze index yielded 40 STs, 40 GTs and 40 intermediates (Figure S2 A). The latter were excluded from the categorical but included in the dimensional analyses. The CR expression became more pronounced in the second hemiblock of the task for both the CS+ $(b=-0.13, S E=0.07, t=-2.01, p=0.05)$ and the CS- $(b=-0.15, S E=0.07, t=-2.13, p=0.04)$, and there was a significant hemiblock $x$ phenotype interaction $(\mathrm{CS}+\mathrm{b}=0.26, \mathrm{SE}=0.09, \mathrm{t}=2.80, \mathrm{p}=$ 0.006; CS-: $\mathrm{b}=0.31, \mathrm{SE}=0.10, \mathrm{t}=3.07, \mathrm{p}<0.002$ ) indicating that enhancement of this $\mathrm{CR}$ as a function of Pavlovian learning was more pronounced in STs than in GTs (Figure S2 B).

\section{Operant Conditioning}

In the operant task, the proportion of responses on the rewarded target did not differ for STs versus GTs ( $\mathrm{p}=0.91$ ), and both groups were significantly above chance (one sample t-test (one-sided): $\left.\mathrm{t}_{(77)}=13.37, \mathrm{p}<0.0005\right)$ indicating operant learning $($ Figure $\mathrm{S} 2 \mathrm{C})$. 


\section{Pavlovian Conditioning}

Categorical analysis: Participants developed a significant subjective preference for the $\mathrm{CS}+$ relative to the $\mathrm{CS}-(\mathrm{b}=1.68, \mathrm{SE}=0.25, \mathrm{t}=6.8, \mathrm{p}<0.0005)$, and this did not differ between STs and GTs defined categorically $(\mathrm{p}=0.72)$ (Figure S2 D).

Participants displayed a pupil dilation response during the CS presentation, ramping up as the time of reward delivery approached. There was a significant decrease in the magnitude of this response as a function of trial $(b=-0.26, \mathrm{SE}=0.07, \mathrm{t}=-4.24, \mathrm{p}<0.0005)$, consistent with a reduction in uncertainty-driven pupil response as the CS-US association is learned, but there was no significant interaction with CS or phenotype $\left(\mathrm{p}_{\mathrm{s}} \geq 0.1\right)$.

\section{Pavlovian Instrumental Transfer}

Pooling data from both hemiblocks, the likelihood of making congruent responses did not differ significantly as a function of CS or phenotype. Separate analyses of the first and second hemiblocks of the task showed a significant CS x phenotype interaction only in the second hemiblock suggesting increased congruent responding in the presence of the CS+ relative to the neutral stimulus in ST and the opposite tendency in GT $(b=-0.31, \mathrm{SE}=0.15, \mathrm{t}=-2.06, \mathrm{p}=0.04)$, although these tendencies were not significant in either STs or GTs in separate group-wise analyses $\left(p_{s}>0.1\right)$, (Figure S2 E). There were no significant main effects of interactions in the model defining ST dimensionally using the gaze index also demonstrated a trend for a CS x gaze index interaction in the second hemiblock $(b=-0.25, \mathrm{SE}=0.13, \mathrm{t}=-1.88, \mathrm{p}=0.06)$, such that participants with lower (more GT-like) values on the gaze index showed greater enhancement of congruent responses in the second hemiblock in the presence of the novel stimulus relative to the 
CS+, with the opposite trend for participants with more ST-like values. The rate of congruent responses in the first hemiblock did not differ significantly as a function of CS or phenotype.

\section{Decision Making}

Categorical analysis: Consistent with the previously reported findings (Cherkasova et al., 2018), there was a significant effect of cues on the propensity to choose riskier prospects (associated with lower reward probabilities) on the Vancouver Gambling Task in the categorical analysis (STs: $\mathrm{n}=40 ; \mathrm{GTs}: \mathrm{n}=40, \mathrm{~b}=-0.71, \mathrm{SE}=0.32, \mathrm{t}=-2.23, \mathrm{OR}[95 \% \mathrm{CI}]=0.49[0.26,0.92], \mathrm{p}=$ 0.03). STs and GTs did not differ significantly in their propensity to take risks $(\mathrm{p}=0.27)$ or to take more risks in the presence of cues (phenotype $\mathrm{x}$ cues: $\mathrm{p}=0.26$ ) (Figure S2 F). In addition, there was a significant effect of trial repetition $(b=0.07, \mathrm{SE}=0.02, \mathrm{t}=4.05, \mathrm{OR}[95 \% \mathrm{CI}]=1.08$ $[1.04,1.11], \mathrm{p}<0.0005)$ : choices became riskier with successive trials.

Dimensional analysis covariates: The results of the dimensional model are reported in the main manuscript. In addition to these main findings, there was a significant effect of trial repetition $(\mathrm{b}=0.07, \mathrm{SE}=0.02, \mathrm{t}=4.44, \mathrm{OR}[95 \% \mathrm{CI}]=1.07[1.04,1.1], \mathrm{p}<0.0005)$ : choices became riskier with successive trails. There was also a significant effect of the task order covariate $(b=-0.39, \mathrm{SE}=0.18, \mathrm{t}=-2.24, \mathrm{OR}[95 \% \mathrm{CI}]=0.68[0.43,1.01], \mathrm{p}=0.03)$ : participants who performed the VGT second made riskier choices.

\section{Analyses Excluding an Influential Observation}

In the Study 1 Vancouver Gambling Task models, one influential observation (DF-Betas $\leq-.63$ for the effect of cues and $\leq-0.48$ for the phenotype (or gaze index) $\mathrm{x}$ cues interaction; Cook's distances $\geq 0.12$ ) also changed the significance of these predictors. Below are the results of the models excluding this influential observation. In the categorical analysis with STs and 
GTs defined using a tertiary split, there was a trend for the effect of cues on the propensity to choose riskier prospects $(b=-0.52, \mathrm{SE}=0.29, \mathrm{t}=-1.79, \mathrm{p}=0.07)$. There was also a trend for $\mathrm{STs}$ to be more risk-prone than GTs $(b=0.48, \mathrm{SE}=0.27, \mathrm{t}=1.82, \mathrm{p}=0.07)$. In the dimensional model, there was a significant effect of cues $(b=-0.60, S E=0.21, t=-2.92, \mathrm{OR}[95 \% \mathrm{CI}]=0.55[0.26$, 0.92], $\mathrm{p}=0.003)$ and a significant phenotype $\mathrm{x}$ cues $\mathrm{x}$ EVR interaction $(b=1.83, \mathrm{SE}=0.93$, $\mathrm{t}=1.97, \mathrm{OR}[95 \% \mathrm{CI}]=6.28[0.56,24.84], \mathrm{p}=0.05)$ suggesting that cues promoted riskier choice more in GTs than in STs in a value-dependent manner, with a greater risk enhancement for prospects with higher expected values.

\section{Study 2}

Sign-Tracking and Goal-Tracking

A tertiary split on the gaze index yielded 19 STs, 19 GTs and 18 intermediates (Figure S3 A). The CR to the CS+ became significantly more pronounced in the second hemiblock for the STs and marginally more pronounced for the GTs (STs: $b=0.29, \mathrm{SE}=0.09, \mathrm{t}=3.14, \mathrm{p}=0.003$, GTs: $b=-0.19, \mathrm{SE}=0.11, \mathrm{t}=-1.82, \mathrm{p}=0.08)$; there was no significant enhancement of $\mathrm{CR}$ to the CS- as a function of hemiblock ( $\mathrm{p} \geq 0.16$ ) (Figure $\mathrm{S} 3 \mathrm{~B}$ ).

\section{Operant Conditioning}

As in Study 1, the proportion of responses on the rewarded target did not differ for STs versus GTs $(\mathrm{p}=0.62)$, and both groups were significantly above chance (one-sided one sample ttest: $\left.\mathrm{t}_{(37)}=8.58, \mathrm{p}<0.0005\right)$ indicating operant learning $($ Figure S3 C).

\section{Pavlovian Conditioning}


Participants developed a significant subjective preference for the CS+ relative to the CS$(b=1.16, S E=0.32, t=3.58, p<0.0006)$, and this did not differ between STs and GTs $(p=0.91)$ (Figure S3 D). Participants also rated the CS+ as significantly more likely to predict rewards than the CS- $(b=9.58, S E=0.98, t=9.80, p<0.0005)$, and this did not differ as a function of phenotype $(\mathrm{p}=0.31)$ (Figure S3 E). In addition, there was a trend for STs to consider both CS more likely to predict rewards $(b=1.88, \mathrm{SE}=0.98, \mathrm{t}=1.92, \mathrm{p}=0.06)$.

As in Study 1, participants displayed a pupil dilation response during the CS presentation, ramping up as the time of reward delivery approached. There was a significant decrease in the magnitude of this response as a function of trial $(b=-0.37, S E=0.10, t=-3.77, p<0.0005)$, which is consistent with a reduction in an uncertainty-driven pupil response as a function of learning the CS-US association. A main effect of phenotype indicated that STs displayed greater anticipatory pupil dilation $(b=0.21, S E=0.10, t=2.17, p=0.04)$. There were no other significant main effects or interactions. Non-significant trends for a main effect of $C S(b=0.18, S E=0.10$, $\mathrm{t}=1.79, \mathrm{p}=0.08)$, a CS $x$ phenotype interaction $(\mathrm{b}=-0.29, \mathrm{SE}=0.15, \mathrm{t}=-2.02, \mathrm{p}=0.051)$, and a CS

$\mathrm{x}$ trial interaction $(\mathrm{b}=0.12, \mathrm{SE}=0.06, \mathrm{t}=1.89, \mathrm{p}=0.059)$ suggested, respectively, that pupil tended to dilate more in anticipation of the CS+ than the CS-, that this occurred preferentially in GTs, and that there tended to be a greater reduction in the anticipatory pupil dilation for the CS+ than the CS- as a function of trial.

\section{Pavlovian Instrumental Transfer}

ST and GT did not differ significantly in the expression of PIT collapsing across hemiblocks $\left(\mathrm{p}_{\mathrm{s}} \geq 0.24\right)$. Separate analyses of the first and second hemiblocks of the task showed a significant CS x phenotype interaction only in the second hemiblock suggesting increased congruent responding in the presence of the CS+ relative to the CS- in ST and the opposite 
tendency in GT $(b=-0.34, S E=0.20, t=-1.68, p=0.1)$, (Figure S3 F). Sign-tracking defined dimensionally as gaze index did not predict a differential response to the CS+ relative to the CSor the neutral stimulus either collapsing across hemiblocks $\left(\mathrm{p}_{\mathrm{s}} \geq 0.55\right)$ or examining them separately $\left(\mathrm{p}_{\mathrm{s}} \geq 0.21\right)$.

\section{Decision Making}

Categorical analysis: There was a significant main effect of cues $(b=-0.46, S E=0.18, t=-$ 2.53, OR $[95 \% \mathrm{CI}]=0.63[0.44,0.90], \mathrm{p}=0.01)$ and a significant cues $\mathrm{x}$ EVR interaction (cues: $\mathrm{b}=-0.43, \mathrm{SE}=0.19, \mathrm{t}=-2.29, \mathrm{OR}[95 \% \mathrm{CI}]=0.65[0.45,0.94], \mathrm{p}=0.02)$. Cues promoted riskier choice preferentially for prospects with higher expected values. This value-dependent riskpromoting effect differed as a function of phenotype (phenotype x cues x EVR: $b=-0.54$, $\mathrm{SE}=0.26, \mathrm{t}=2.13, \mathrm{OR}[95 \% \mathrm{CI}]=1.72[1.04,2.84], \mathrm{p}=0.03)$. Separate post-hoc models in STs and GTs showed that only the GTs (cues x EVR: $b=-0.52, \mathrm{SE}=0.19, \mathrm{t}=-2.69, \mathrm{OR}[95 \% \mathrm{CI}]=$ $0.59[0.41,0.87], \mathrm{p}=0.007$ ) and not the STs (cues x EVR: $\mathrm{p}=0.37$ ) significantly increased their risk taking in response to the cues (Figure S3 G).

Dimensional analysis covariates: The results of the dimensional model are reported in the main manuscript. In addition to these main findings, there was a significant effect of the task order covariate $(b=0.54, \mathrm{SE}=0.27, \mathrm{t}=2.00, \mathrm{OR}[95 \% \mathrm{CI}]=1.71[1.01,2.89], \mathrm{p}=0.05)$ : participants who performed the uncued VGT first and cued VGT second made riskier choices. 


\section{REFERENCES}

Cherkasova, M. V, Clark, L., Barton, J. J. S., Schulzer, M., Shafiee, M., Kingstone, A., ... Winstanley, C. A. (2018). Win-Concurrent Sensory Cues Can Promote Riskier Choice. Journal of Neuroscience, 38(48), 10362-10370.

Garofalo, S., \& di Pellegrino, G. (2015). Individual differences in the influence of task-irrelevant Pavlovian cues on human behavior. Frontiers in Behavioral Neuroscience, 9(June), 163. https://doi.org/10.3389/fnbeh.2015.00163 


\section{FIGURE LEGENDS}

Figure 1: Pavlovian Instrumental Transfer Tasks

A) Operant paradigm. B) Pavlovian Paradigm. C) Pavlovian Instrumental Transfer (PIT). D)

Heat map used to guide interest area definition. VI = variable interval; ITI = intertrial interval; $\mathrm{CS}=$ conditioned stimulus

Figure 2: Study 1, Pavlovian Conditioning, Operant Conditioning and Pavlovian Instrumental Transfer, and Decision Making for STs and GTs Defined Categorically

A) Gaze index distribution. B) Expression for the sign- and goal-tracking CR as a function of task hemiblock. C) Operant conditioning. D) Subjective liking ratings for CS+ and CS-. E) Pavlovian Instrumental Transfer. F) The effect of cues on decision making as a function of phenotype (sign-trackers and goal-trackers defined categorically). The lines represent predicted probabilities of risky choice; ribbons represent standard error of the mean. ST=sign trackers; GT=goal trackers; IM=intermediates; $\mathrm{CS}+=$ reward-predictive conditioned stimulus; $\mathrm{CS}-=$ nonreward-predictive conditioned stimulus; $\mathrm{N}=$ neutral stimulus. $* * * \mathrm{p}<0.0005$.

Figure 3: Study 2, Pavlovian Conditioning, Operant Conditioning and Pavlovian Instrumental Transfer, and Decision Making for STs and GTs Defined Categorically

A) Gaze index distribution. B) Expression for the sign- and goal-tracking CR as a function of task hemiblock. C) Operant conditioning. D) Subjective liking ratings for CS+ and CS-. E) Reward likelihood ratings for CS+ and CS-. F) Pavlovian Instrumental Transfer. G) The effect of cues on decision making as a function of phenotype (sign-trackers and goal-trackers defined categorically). The lines represent predicted probabilities of risky choice; ribbons represent standard error of the mean. ST=sign trackers; GT=goal trackers; IM=intermediates; $\mathrm{CS}+=$ reward-predictive conditioned stimulus; $\mathrm{CS}-=$ non-reward-predictive conditioned stimulus; $\mathrm{N}=$ neutral stimulus. $* * * \mathrm{p}<0.0005$. 
Figure 2

A Operant Conditioning
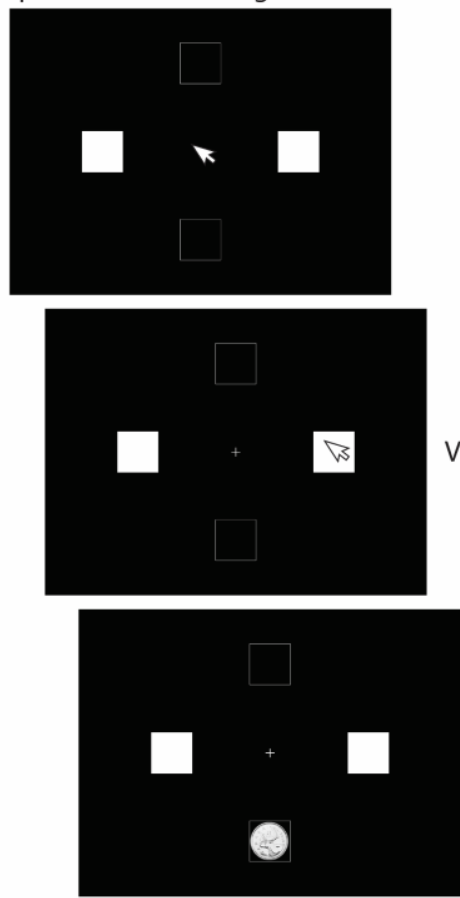

B Pavlovian Conditioning

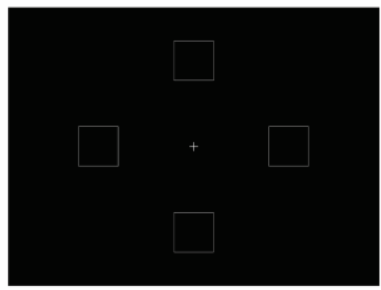

ITI:

$500-4000 \mathrm{~ms}$

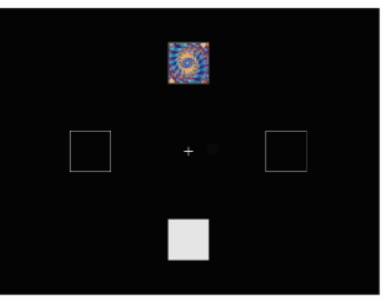

CS+ or CS-

Study 1: $5000 \mathrm{~ms}$

VI: 4-12 s

Reward $500 \mathrm{~ms}$

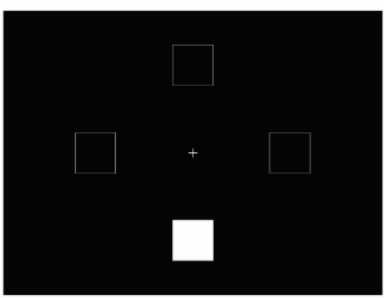

[6]

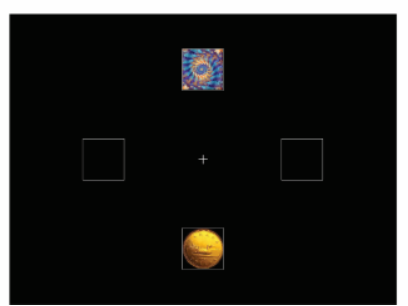

Study 2: $7000 \mathrm{~ms}$

Response $\mathrm{Bm}^{\mathrm{m}}$ (Study 1 only)

Reward
C Pavlovian Instrumental Transfer

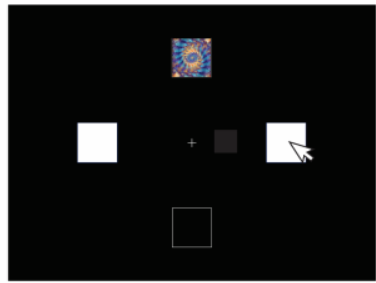

$\mathrm{CS}+$

CS-

CS novel

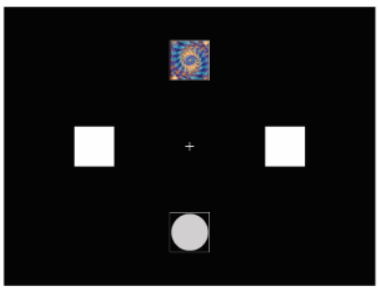

Non-reward (extinction) $500 \mathrm{~ms}$

CS+: $80 \%$; CS-: $0 \%$

Study 1: $1000 \mathrm{~ms}$

D Interest Area Definition

Study 2: $2000 \mathrm{~ms}$

$\mathbb{B}$ response required
Fixation duration based on 1300 trials and 10205 fixations

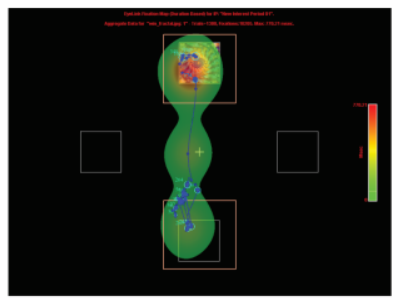


Figure S2

A

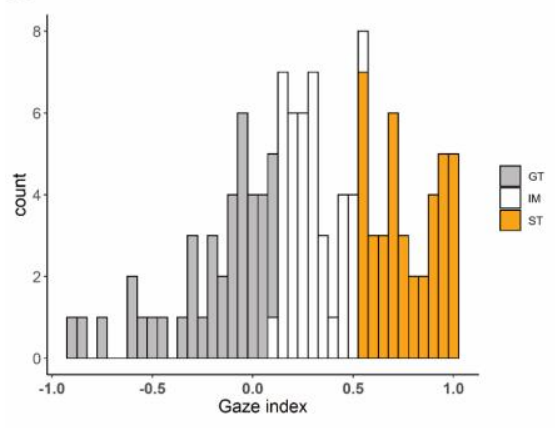

C

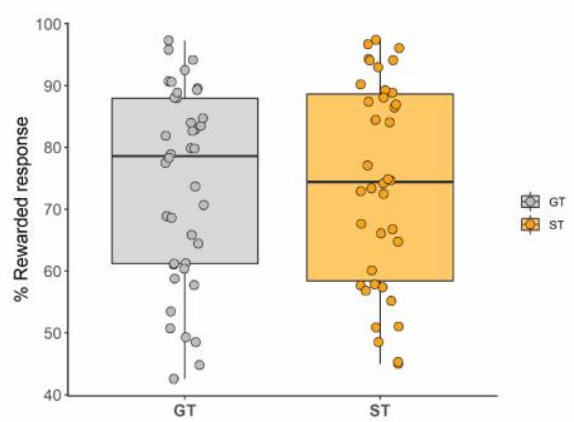

E

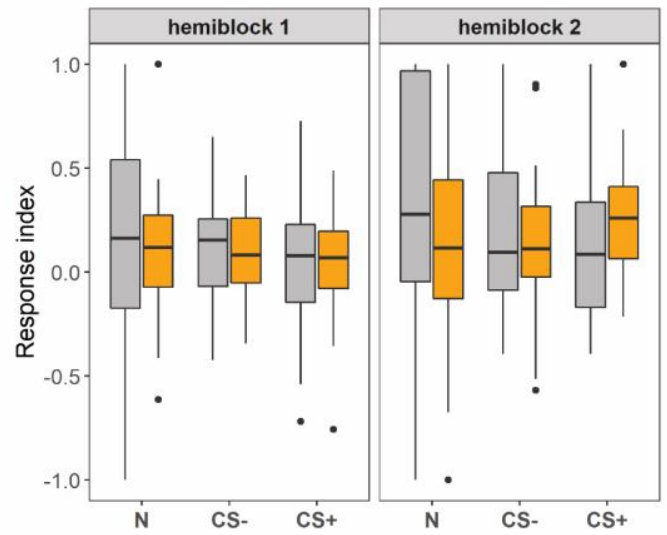

CS+

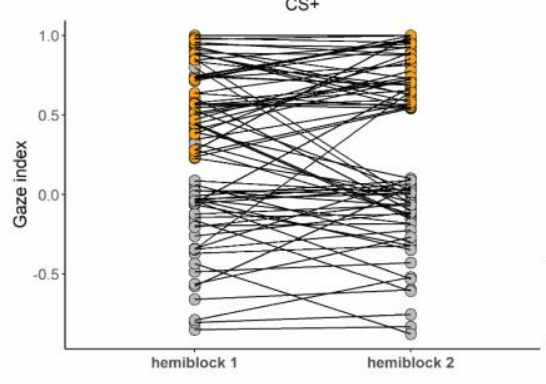

cS-

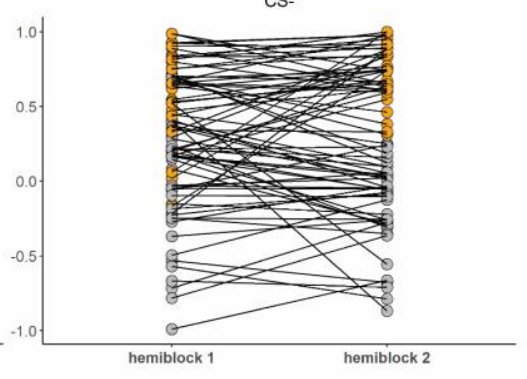

D

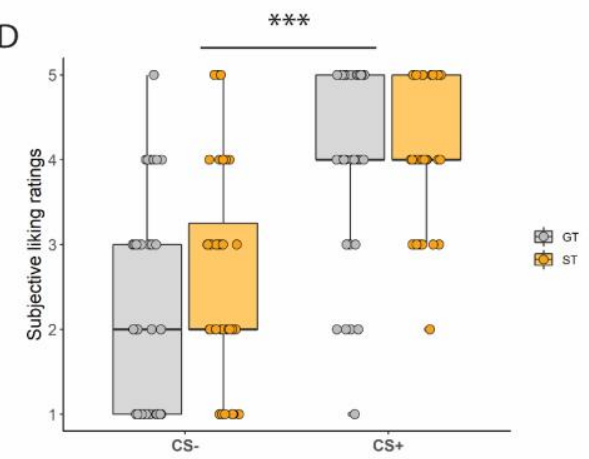

F

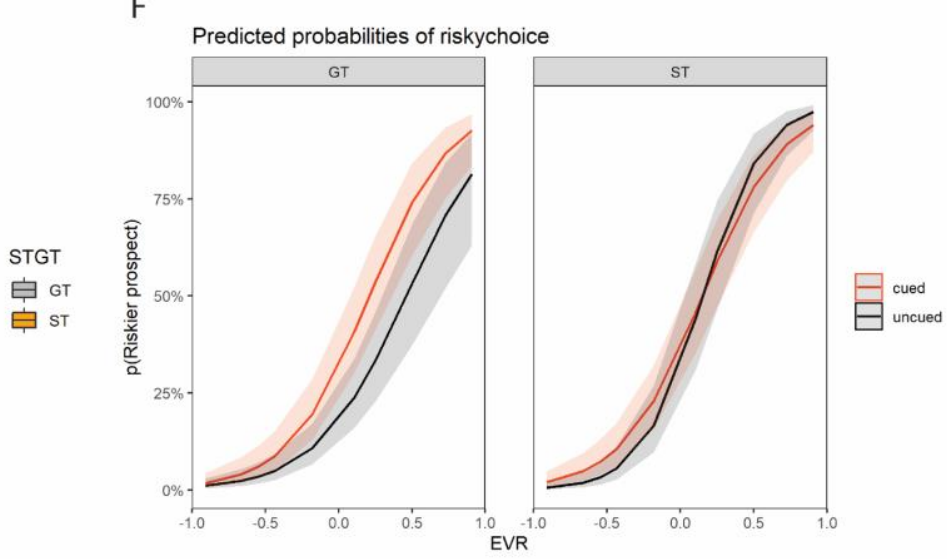


Figure S3

A

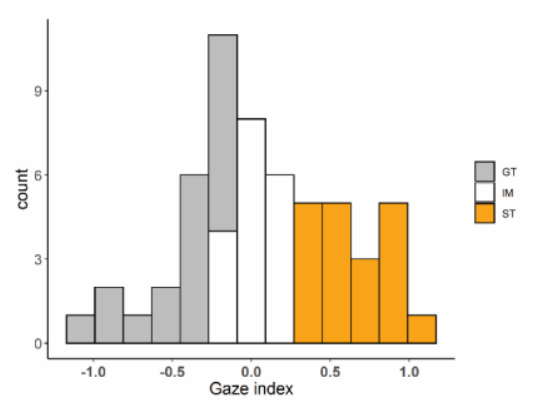

C

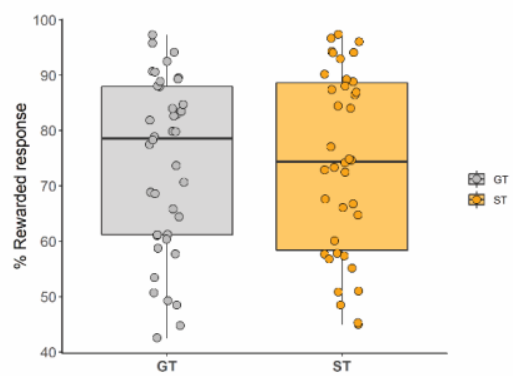

E

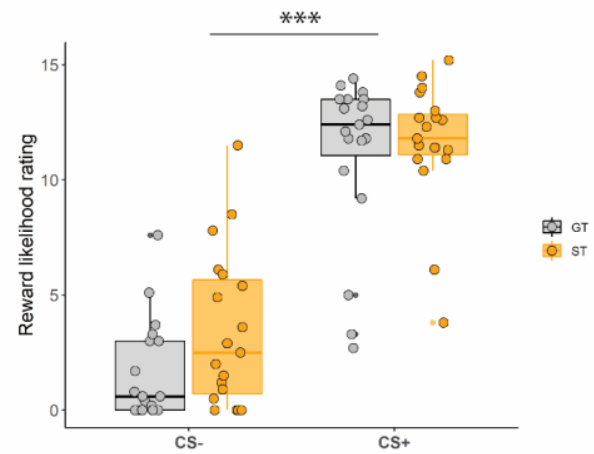

B
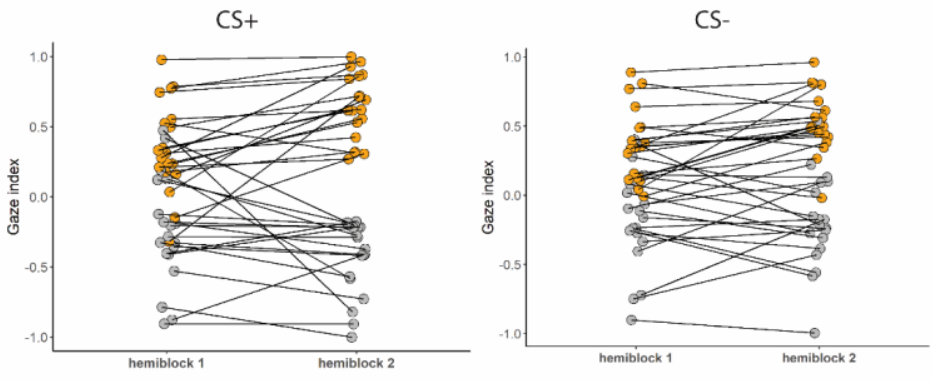

D

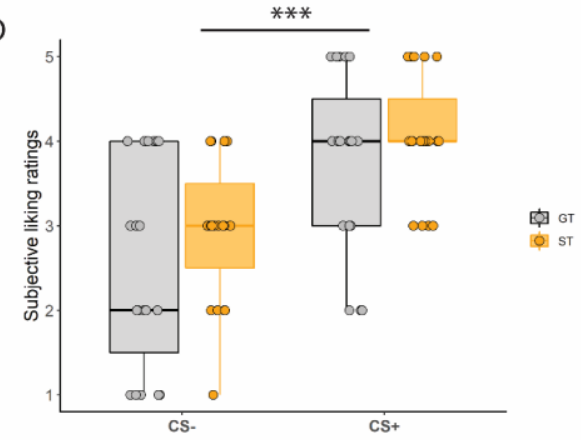

F

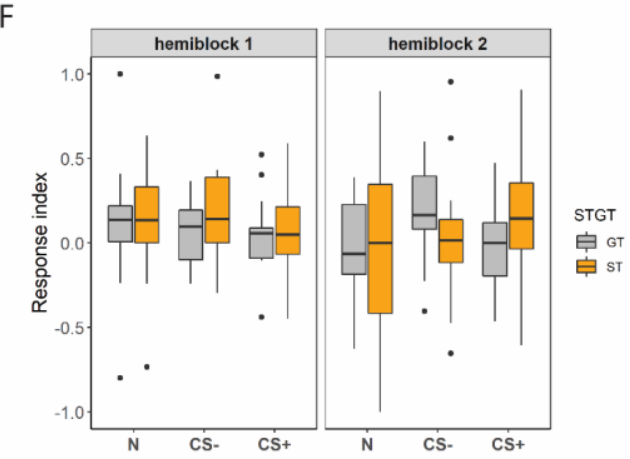

G

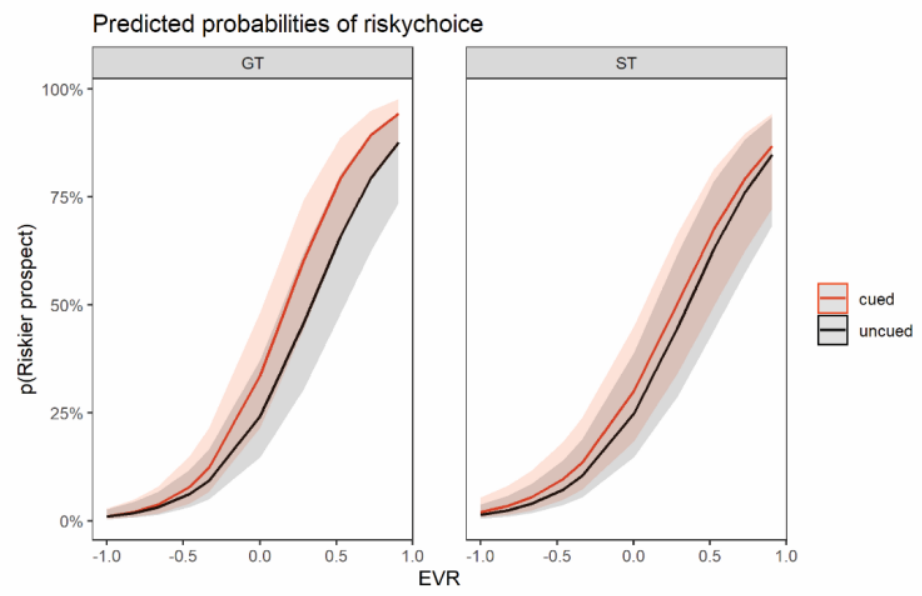

